\title{
HOMOLOGY OF SPACES WITH OPERATORS. II
}

\author{
BY \\ SAMUEL EILENBERG AND SAUNDERS MACLANE(1)
}

\section{INTRODUCTION $\left({ }^{2}\right)$}

The fundamental group $\pi_{1}$ of an arcwise connected topological space determines the one-dimensional homology groups of that space; indeed, the homology group $H_{1}$ formed from finite chains with integral coefficients is simply the fundamental group made abelian. On the next dimension the second homotopy group $\pi_{2}$ has a natural homomorphism $\pi_{2} \rightarrow H_{2}$ into the second integral homology group. The image of $\pi_{2}$ under this homomorplfism is a subgroup $\Sigma_{2}$ of $H_{2}$ which may be described as the group of those homology classes which contain "spherical" cycles. The kernel of this homomorphism is a subgroup $\gamma_{2}$ of $\pi_{2}$ which is known to contain all the elements of $\pi_{2}$ of the form $x s-s$, where $s$ is any element of $\pi_{2}, x$ any element of $\pi_{1}$, and the product $x s$ denotes the result of operating on $s$ with the element $x$. The kernel $\gamma_{2}$ is thus determined in part by the fashion in which the fundamental group $\pi_{1}$ operates on $\pi_{2}$, while the "spherical" homology group $\Sigma_{2}$ is determined as $\pi_{2} / \gamma_{2}$.

In a fundamental paper [9], Hopf has shown that the remaining portion $\mathrm{H}_{2} / \mathrm{\Sigma}_{2}$ of the second homology group is determined by the fundamental group and has described explicitly a method of constructing this factor group from $\pi_{1}$. Thus the second homology group $H_{2}$ is an abelian group extension of an image $\Sigma_{2}$ of $\pi_{2}$ by a group $H_{2} / \Sigma_{2}$ depending only on $\pi_{1}$. Thus one might conjecture that $H_{2}$ is determined by, and can be computed from, the two homotopy groups $\pi_{1}$ and $\pi_{2}$ and the operators of $\pi_{1}$ on $\pi_{2}$. Such a determination would involve two additional items: (i) a complete description of the kernel $\gamma_{2}$ : (ii) a description of the "factor set" for the extension of $\Sigma_{2}$ by $H_{2} / \Sigma_{2}$.

This conjecture is false. To show this, we construct several specific spaces, starting in each case with a 3-cell $E^{3}$ with boundary a 2-sphere $S^{2}$.

First, choose any continuous map $f: S^{2} \rightarrow S^{2}$ of degree 2, and consider the space $X$ obtained from $E^{3}$ by identifying those pairs of points $(x, y)$ on $S^{2}$ for which $f(x)=f(y)$. This space may be seen to be simply connected (that is, $\left.\pi_{1}=0\right)$; hence by a theorem of Hurewicz $\left[12_{2}\right]$ its second homotopy group $\pi_{2}$ is identical with $H_{2}$ (that is, $\gamma_{2}=0$ and $\Sigma_{2}=H_{2}$ ). A suitable cell subdivision shows that the latter is a cyclic group of order 2 . Thus

Presented to the Society, August 23,1948, under the title Determination of the second homology and cohomology groups of a space by means of homotopy invariants; received by the editors November $17,1947$.

(1) John Simon Guggenheim Memorial Fellow.

(2) Paper I of this title is listed as [19] in the bibliography at the end of this paper. A knowledge of [19] is not indispensable for the reading of this paper. 


$$
\pi_{1}(X)=0, \quad \pi_{2}(X)=Z_{2}, \quad H_{2}(X)=Z_{2},
$$

where $Z_{2}$ denotes a cyclic group of order 2 .

Second, let $g: S^{2} \rightarrow P^{2}$ be the natural map of $S^{2}$ onto the projective plane $P^{2}$, as obtained by identifying diametrical points on $S^{2}$. The projective 3space $P^{3}$ may then be obtained from $E^{3}$ by identifying points $x, y$ on $S^{2}$ such that $g(x)=g(y)$. Since the universal covering space of $P^{3}$ is $S^{3}$ one has

$$
\pi_{1}\left(P^{3}\right)=Z_{2}, \quad \pi_{2}\left(P^{3}\right)=0, \quad H_{2}\left(P^{3}\right)=0 .
$$

Third, consider the composite map $h=g f: S^{2} \rightarrow P^{2}$. The map $g: S^{2} \rightarrow P^{2}$ may be regarded as an element of the second homotopy group $\pi_{2}\left(P^{2}\right)$ and is in fact a generator of the latter group (which is infinite cyclic). Since $f$ is of degree 2, the map $h$, regarded as an element of $\pi_{2}\left(P^{2}\right)$, is twice the generator of $\pi_{2}\left(P^{2}\right)$. Let $Y$ be the space obtained from $E^{3}$ by identifying pairs $x, y$ of $S^{2}$ such that $h(x)=h(y)$. Then

$$
\pi_{1}(Y)=Z_{2}, \quad \pi_{2}(Y)=Z_{2}, \quad H_{2}(Y)=0 .
$$

Finally, construct the cartesian product $Y^{\prime}=X \times P^{3}$. The results (1) and (2), combined with general rules for computing homology and homotopy groups of product spaces, yield

$$
\pi_{1}\left(Y^{\prime}\right)=Z_{2}, \quad \pi_{2}\left(Y^{\prime}\right)=Z_{2}, \quad H_{2}\left(Y^{\prime}\right)=Z_{2} .
$$

In all these spaces the fashion in which $\pi_{1}$ operates on $\pi_{2}$ must be trivial or "simple," in the sense that $x s=s$ for all $s \in \pi_{2}, x \in \pi_{1}$. Indeed, $\pi_{2}$ is at the worst cyclic of order 2 , hence can have no automorphisms $s \leftrightarrow x s$ other than the identity. A comparison of the displays (3) and (4) now indicates that the spaces $Y$ and $Y^{\prime}$ as constructed do have the same first and second homotopy groups and the same operators of the one upon the other, but have different second homology groups. In a word, $H_{2}$ is not determined by $\pi_{1}$ and $\pi_{2}$.

It is none the less possible to determine $\mathrm{H}_{2}$ from these homotopy groups and a certain supplemental homotopy invariant. Our prime objectives are the introduction of this new homotopy invariant $\boldsymbol{k}^{3}$, the demonstration that this invariant, together with $\pi_{1}, \pi_{2}$, and the operators of $\pi_{1}$ on $\pi_{2}$, suffices to determine $H_{2}$, and the derivation of the explicit algebraic formulas which give the second homology group in terms of these homotopy quantities.

The new invariant is essentially a function $k^{3}(x, y, z)$ of three variables $x, y, z$ in the (multiplicative) group $\pi_{1}$, taking values in the (additive) group $\pi_{2}$. Its geometric definition follows a scheme used by Robbins [25] in the classification of the maps of a 2-complex into a space. This geometric definition involves a number of arbitrary choices; in consequence, the function $k^{3}$ is not unique. It may be modified by the addition of any function $\delta h^{2}(x, y, z)$ which is obtained from a function $h^{2}(x, y)$ of two arguments in $\pi_{1}$, with values in $\pi_{2}$, by the algebraic formula 


$$
\delta h^{2}(x, y, z)=x h^{2}(y, z)-h^{2}(x y, z)+h^{2}(x, y z)-h^{2}(x, y) .
$$

(The first term in this formula involves the element $x \in \pi_{1}$ operating on the element $h^{2}(y, z) \in \pi_{2}$.) Modulo alterations by such functions $\delta h^{2}$, the function $k^{3}$ is unique. Furthermore, it always satisfies an identity $\delta k^{3}=0$, where $\delta k^{3}$ is a function of four variables in $\pi_{1}$ and is defined from $k^{3}$ by a formula

$$
\begin{aligned}
\delta k^{3}(x, y, z, t)= & x k^{3}(y, z, t)-k^{3}(x y, z, t)+k^{3}(x, y z, t) \\
& -k^{3}(x, y, z t)+k^{3}(x, y, z) .
\end{aligned}
$$

The class of all functions $k^{3}$ thus obtained is denoted by $\boldsymbol{k}^{3}$.

In the case of the two spaces used for our illustration in (3) and (4) above, we may write $\pi_{1}=Z_{2}$ as the multiplicative group of the numbers \pm 1 and $\pi_{2}=Z_{2}$ as the additive group of integers modulo 2; the functions $k$ and $k^{\prime}$ of the spaces $Y$ and $Y^{\prime}$ then turn out to be

$$
k(-1,-1,-1) \equiv 1, \quad k(x, y, z) \equiv 0(\bmod 2)
$$

for any $(x, y, z) \neq(-1,-1,-1)$ in $\pi_{1}$, and

$$
k^{\prime}(x, y, z) \equiv 0 \quad(\bmod 2), \quad x, y, z \text { in } \pi_{1} .
$$

This result can be deduced readily from the explicit algebraic formulas ( $\$ 12)$ by which we determine $H_{2}$ in terms of $\pi_{1}, \pi_{2}$, and $k^{3}$. It can also be shown that no modification of the $k$ of (7) by a function $\delta h^{2}$ will yield the $k^{\prime}$ of (8). Hence the invariants $k^{3}$ of the spaces $Y$ and $Y^{\prime}$ are in fact distinct, and it is this difference which accounts for the differing second homology groups exhibited in (3) and (4).

The identities (5) and (6) appear in other connections. In these formulas the arguments $x, y, z$, and so on, may be taken from any multiplicative group $W$ (instead of $\pi_{1}$ ), and the values from any abelian group $G$ (instead of $\pi_{2}$ ) on which $W$ acts as a group of automorphisms. The set of all functions $f^{8}$ of three variables from $W$ to $G$ which satisfy the identity $\delta f^{3}=0$ constitute a group of "three-dimensional cocycles" which contains the group of all those functions ("three-dimensional coboundaries") which have the form $f^{8}=\delta h^{2}$ for some function $h^{2}$ of two variables on $W$ to $G$. The factor group (cocycles modulo coboundaries) is called the third cohomology group $H^{3}(W, G)$ of $W$ with coefficients in $G$. Similar cohomology groups of the group $W$ can be defined for other positive dimensions [6, 7]; for example, the group $H^{2}(W, G)$ is obtained from functions $h^{2}$ with $\delta h^{2}=0$; this identity on $h^{2}$ is precisely the identity for the "factor sets" $h^{2}$ in the purely algebraic study of the group extensions of $G$ by $W$.

In this language, the new invariant of the space is best described not as a single function or cocycle $k^{3}$, but as the cohomology class

$$
k^{2} \in H^{3}\left(\pi_{1}, \pi_{2}\right)
$$


containing this cocycle; this class is then independent of any choices made in the definition of the function. In particular, in the example $\pi_{1}=\pi_{2}=Z_{2}$, the group $H^{3}\left(\pi_{1}, \pi_{2}\right)$ is cyclic of order 2 , and the cohomology classes of $k$ and $k^{\prime}$ in (7) and (8) are precisely the two elements of the group $H^{3}$.

The technique used to find the effect of the groups $\pi_{1}$ and $\pi_{2}$ on homology groups applies equally well if the homotopy groups $\pi_{1}$ and $\pi_{q}$ are given, with $\pi_{i}=0$, for $1<i<q$. Such a space is said to be aspherical in dimensions less than $q$. Hurewicz $\left[12_{4}\right]$ showed that such aspherical spaces with isomorphic fundamental groups have isomorphic homology groups in dimensions less than $q$. An algebraic formula for calculating the second homology group $H_{2}$ from the fundamental group in the case $q>2$ was given by Hopf [9] who simultaneously showed that his formula gives $H_{2} / \Sigma_{2}$ in the case $q=2$. This formula is expressed in terms of a representation of $\pi_{1}$ by generators and relations. A more invariant form can be obtained by considering instead the cohomology groups, from which the homology groups may be found by duality. For example, if $q>2$, Hopf's result becomes the assertion that the second cohomology group $H^{2}(X, G)$ of the space $X$ with coefficients in any abelian group $G$ is isomorphic to the (algebraic!) second cohomology group $H^{2}\left(\pi_{1}(X), G\right)$ of the fundamental group $\pi_{1}(X)$ of that space. For higher dimensions, the authors [5] and Eckmann [1] found the corresponding result that $H^{n}(X, G)$ is isomorphic to $H^{n}\left(\pi_{1}(X), G\right)$ for $n<q$, and that $H^{q}(X, G)$ has a subgroup, isomorphic to $H^{q}\left(\pi_{1}(X), G\right)$. Similar results, expressed directly in terms of homology groups, have been found by Hopf [10] and by Freudenthal [8].

Our present results give a complete determination of the $q$ th cohomology group $H^{q}$ for these spaces aspherical in dimensions less than $q$. In addition to the homotopy groups $\pi_{1}$ and $\pi_{q}$ these spaces have a homotopy invariant $\boldsymbol{k}^{q+1}$ in $H^{q+1}\left(\pi_{1}, \pi_{q}\right)$ analogous to the invariant $\boldsymbol{k}^{3}$ introduced above. Using $k^{q+1}$ we obtain an explicit algebraic formula for the cohomology group $H^{q}(X, G)$ of the space.

This result, if proved by a direct analysis in the space $X$ as outlined in our note [21], requires a considerable number of homotopy arguments, as for instance in the proof of the general identity $\delta k^{q+1}=0$. Alternatively, following a line of procedure already employed by Hopf [10], Eckmann $[1,27]$, and Eilenberg [19], one can replace the space $X$ by its universal covering space $\tilde{X}$. All the quantities which concern us may then be translated [19] to this covering space. Thus the fundamental group $\pi_{1}$ becomes a group of operators (covering transformations) on $\tilde{X}$, the group $\pi_{q}$ becomes the $q$ th homology group of $\tilde{X}$, the $q$ th cohomology group of $X$ becomes a special cohomology group of $\tilde{X}$ constructed from cocycles which are "equivariant" [19] under the covering transformations of $\pi_{1}$, and the invariant $k^{q+1}$ reappears as a certain type of obstruction $1^{a+1}$. When the space is replaced by its singular complex, the problem now becomes a topic in the theory of complexes with a group of operators (cf. de Rham [15]). We can call on the first 
paper [19] of this title for the theory of the equivariant groups which is then needed. The main theorem now becomes the assertion that in a space with a fixed-point-free group $W$ of operators, acyclic in dimensions less than $q$, the qth "equivariant" cohomology groups are determined by the group of operators, by the $q$ th homology group $H_{q}$, and by a certain "obstruction" invariant $1^{q+1} \in H^{q+1}\left(W, H_{q}\right)$. This result is of interest in its own right, in that it applies to spaces which need not have arisen as universal covering spaces.

Our first chapter considers complexes with operators and the obstructions $1^{q+1}$ to the extensions of certain chain transformations between such complexes. The fundamental theorem is that of $\$ 7$; it affords a comparison between any two complexes with "free" operators from a group $W$, both acyclic up to dimension $q$, and both possessing the same obstruction.

The second chapter gives the basic group construction (\$12) of a cohomology group from $\pi_{1}, \pi_{q}$, and $1^{q+1}$, viewed as a construction for any groups $W\left(=\pi_{1}\right)$ and $H\left(=\pi_{q}\right)$ and an element $l^{q+1} \in H^{q+1}(W, H)$. This construction yields the $q$ th equivariant cohomology group of a complex with free operators $W$, which has $l^{q+1}$ as its "obstruction." This basic fact is proved in two different ways.

The third chapter returns to the original problem of a space $X$ aspherical in dimensions less than $q$, and uses the singular homology theory of [3] and the known relation between $X$ and its covering space to translate the result of the previous chapter. This gives the main theorem. By considering the case of a coefficient group $G$ with $W$ as a group of operators, we obtain by the same technique a more general theorem for the cohomology groups with local coefficients, in the sense of Steenrod [17]. The fundamental theorem is stated in full as Theorem 20.1, and the theorem for local coefficients as Theorem 22.1.

The fourth chapter applies these results to lens spaces and to the generalized lens spaces in the sense of de Rham [24]. We calculate the invariant $\boldsymbol{k}^{a+1}$ for these spaces and connect this result with certain known facts about such spaces. Since these lens spaces have a cyclic fundamental group, this chapter requires an explicit description $(\$ 26)$ of the cohomology group $H^{q}$ of a cyclic group.

In case a space $X$ has $\pi_{m}$ and $\pi_{q} \neq 0$, with $\pi_{i}=0$ for $1 \leqq i<m$ and $m<i<q$, a similar technique (cf. [5, chap. IV]) will determine the $q$ th cohomology and homology groups of the space in terms of $\pi_{m}, \pi_{q}$, and a suitable algebraic cohomology class resembling $k^{q+1}$. For this case the device of the universal covering space is not applicable, so that the argument must be made directly in terms of the homotopy groups. The details will be treated in a later paper.

\section{Chapter I. Characteristic cocycles}

1. Complexes with operators. We consider throughout complexes $K$ with operators $W$. Here $W$ is a given multiplicative group, $K$ is an abstract closure- 
finite cell complex (in the usual sense; cf. $[19, \S 1]$ ), and $W$ operates on the left as a group of chain transformations of $K$. The latter means that every element $w \in W$ determines an automorphism $c \rightarrow w c$ of the group $C_{q}(K)$ of finite integral $q$-chains of $K$, for each dimension $q$, in such fashion that

$$
\partial w c=w \partial c, \quad w_{1}\left(w_{2} c\right)=\left(w_{1} w_{2}\right) c, \quad 1 c=c,
$$

for every $c \in C_{q}(K)$, and for $w, w_{1}, w_{2} \in W$.

The complex $K$ with operators will be assumed throughout to be augmentable. If $J\left(c_{0}\right)$ denotes the sum of the coefficients in the finite integral 0 -chain $c_{0}$ of $K$, this requires the following three properties for $K$ and $W$ :

(i) $K$ has no cells of negative dimension and at least one cell of dimension 0 ;

(ii) $J\left(\partial c_{1}\right)=0$ for any finite integral 1 -chain $c_{1}$ of $K$;

(iii) $J\left(w c_{0}\right)=J\left(c_{0}\right)$ for each $w \in W$ and $c_{0} \in C_{0}(K)$.

Conditions (i) and (ii) assert that the cell complex $K$ itself is augmentable; it can indeed be augmented by using the additive group of integers as the $(-1)$-chains and the mapping $J: C_{0}(K) \rightarrow$ integers as the corresponding boundary homomorphism. Condition (i) asserts that this homomorphism is onto, and condition (ii) that $J \partial=0$. Condition (iii) requires that the operators are augmentable, in that $\partial w=w \partial$ still holds in the augmented complex, provided that each $w$ operates as the identity automorphism of the group of integers.

In $K$ the group $C_{q}(K)$ of finite chains with integral coefficients contains the usual groups $Z_{q}(K)$ and $B_{q}(K)$ of cycles and boundaries, respectively, and leads to the homology group $H_{q}(K)=Z_{q}(K) / B_{q}(K)$. We denote by

$$
\eta: Z_{q}(K) \rightarrow H_{q}(K)
$$

the natural homomorphism (with kernel $B_{q}$ ) of cycles into homology classes. In addition to $H_{0}(K)$ we introduce the reduced 0 th homology group $\bar{H}_{0}(K)$, obtained by considering a 0 -chain $c_{0}$ to be a reduced cycle if and only if $J\left(c_{0}\right)=0$; thus $\bar{H}_{0}(K)$ is the 0 th homology group of the augmented complex. The conditions (1.1) and (iii) show that the chain transformations of $W$ induce automorphisms $w: H_{q}(K) \rightarrow H_{q}(K), w: \bar{H}_{0}(K) \rightarrow \bar{H}_{0}(K)$; hence $W$ may be regarded as a group of left operators for the homology groups $H_{q}(K)$ and $\bar{H}_{0}(K)$.

The augmentable complex $K$ (with operators $W$ ) is acyclic in dimensions less than $q, q>0$, if the homology groups of finite chains in $K$ with integral coefficients vanish in the relevant dimensions:

$$
\bar{H}_{0}(K)=0, \quad H_{i}(K)=0, \quad 0<i<q .
$$

If $A, B$ are any abelian groups with left operators $W$, an equivariant or operator homomorphism $\alpha: A \rightarrow B$ is a homomorphism such that $w \alpha=\alpha w$ for all $w \in W$. In particular, given the complex $K$ with operators $W$ and a (topo- 
logical) abelian group $G$ with $W$ as left operators, the group $C_{e}^{q}(K, G)$ of equivariant cochains is simply the group of all operator homorphisms $f: C_{q}(K)$ $\rightarrow G$. The coboundary $\delta f$ of $f$ is an operator homomorphism $\delta f=f \partial: C_{q+1}(K) \rightarrow G$. These definitions lead in the usual fashion to the groups $Z_{e}^{q}$ and $B_{e}^{q}$ of equivariant cocycles and coboundaries and to the equivariant cohomology groups $H_{e}^{q}(K, G)=Z_{e}^{q} / B_{e}^{q}[19, \S 4]$.

2. Free operators. Let the multiplicative group $W$ operate (on the left) on the additive abelian group $F$. We shall say that $W$ operates freely on $F$ if there is a collection $\left\{x_{\mu}\right\}$ of elements of $F$ such that the elements $w x_{\mu}$, for all $w$ in $W$, constitute a set of free generators for the free group $F$; the collection $\left\{x_{\mu}\right\}$ itself will be called a $W$-base. As in the case of ordinary free groups, we have the following two characteristic properties for groups with free operators.

LEMma 2.1. If $W$ operates freely on the abelian group $F$ and if $\alpha: A \rightarrow C$ is an operator homomorphism of $A$ onto $C$, where $A$ and $C$ are abelian groups with operators $W$, then every operator homomorphism $f: F \rightarrow C$ can be "lifted" to an operator homomorphism $f^{\prime}: F \rightarrow A$ in such fashion that $\alpha f^{\prime}=f$.

Proof. Choose a $W$-base $\left\{x_{\mu}\right\}$ of $F$. Since $\alpha$ maps $A$ onto $C, f x_{\mu}$ has the form $\alpha a_{\mu}$ for some $a_{\mu} \in A$. The transformation $f^{\prime}$ with $f^{\prime}\left(w x_{\mu}\right)=w a_{\mu}$, extended by linearity to all of $F$, is the required homomorphism.

LEMMA 2.2. If $W$ operates on an abelian group $H$, there exists a group $F$ on which $W$ operates freely and an operator homomorphism $\eta_{0}: F \rightarrow H$ of $F$ onto $H$.

Proof. Construct the abelian free group $F$ with generators $(w, h)$ for all pairs $w \in W, h \in H$, set $u(w, h)=(u w, u h)$ for any $u \in W$ and $\eta_{0}(w, h)=h$, and extend these mappings by linearity to obtain operators $u$ on $F$ and a homomorphism $\eta_{0}: F \rightarrow H$. A $W$-base of $F$ is then the set $\{(1, h)\}$, and $\eta_{0}$ maps $F$ onto $H$.

If $K$ is a complex with operators $W$, then we say that $W$ operates freely on $K$ provided that $W$ operates freely on each of the groups $C_{\boldsymbol{i}}(K)$ of finite integral chains, for all dimensions $i$.

3. Chain transformations and chain homotopy. Let $K_{1}, K_{2}$ be two augmentable complexes with the same group $W$ of operators. For any integer $q>0$, the $q$-skeleton $K_{i}^{q}$ consists of all cells of $K_{i}$, of dimensions not greater than $q$, with the same operators $W$; it is again an augmentable complex with operators $W$. An equivariant chain transformation

$$
\alpha: K_{1}^{q} \rightarrow K_{2}^{q}
$$

of these $q$-skeletons is a set of operator homomorphisms

$$
\alpha_{i}: C_{i}\left(K_{1}\right) \rightarrow C_{i}\left(K_{2}\right), \quad i=0, \cdots, q,
$$

such that 


$$
\partial \alpha_{i}=\alpha_{i-1} \partial
$$$$
i=1, \cdots, q
$$

and

$$
J\left(\alpha_{0} c_{0}\right)=J\left(c_{0}\right),
$$

$c_{0} \in C_{0}\left(K_{1}\right)$;

the second condition is the requirement that $\alpha$ be augmentable. In the usual fashion, each such $\alpha$ induces operator homomorphisms,

$$
\alpha_{0}: \bar{H}_{0}\left(K_{1}\right) \rightarrow \bar{H}_{0}\left(K_{2}\right), \quad \alpha_{i}: H_{i}\left(K_{1}\right) \rightarrow H_{i}\left(K_{2}\right), \quad i=0, \cdots, q-1,
$$

of the integral homology groups. In these definitions, one may also set $q=\infty$; then $\alpha_{i}$ must be defined and (3.1) must hold for all integers $i$.

Two equivariant chain transformations $\alpha: K_{1}^{q^{\prime}} \rightarrow K_{2}^{q^{\prime}}$ and $\beta: K_{1}^{\alpha^{\prime \prime}} \rightarrow K_{2}^{q^{\prime \prime}}$, defined for dimensions $q^{\prime} \geqq q$ and $q^{\prime \prime} \geqq q$, are chain homotopic in dimensions not greater than $q$ if there exists a sequence of operator homomorphisms

$$
D_{i}: C_{i}\left(K_{1}\right) \rightarrow C_{i+1}\left(K_{2}\right), \quad i=0, \cdots, q,
$$

such that, with the usual conventions for the addition of homomorphisms,

$$
\beta_{i}=\alpha_{i}+\partial D_{i}+D_{i-1} \partial,
$$$$
i=0, \cdots, q \text {. }
$$

(For $i=0$, the term $D_{i-1} \partial$ is to be omitted.) We write

$$
D: \alpha \simeq \beta(\operatorname{dim} \leqq q)
$$

or simply $\alpha \simeq \beta$ (dim $\leqq q$ ) to indicate this homotopy. Under these circumstances $\alpha$ and $\beta$ induce the same homomorphisms of the integral homology groups $\bar{H}_{0}$ and $H_{i}$, for $0 \leqq i<q$. The relation $\alpha \simeq \beta$ (dim $\left.\leqq q\right)$ of homotopy is manifestly reflexive, symmetric, and transitive; in particular, the transitivity follows from the rule that $D: \alpha \simeq \beta(\operatorname{dim} \leqq q)$ and $D^{\prime}: \beta \simeq \gamma(\operatorname{dim} \leqq q)$ imply $D+D^{\prime}: \alpha \simeq \gamma(\operatorname{dim} \leqq q)$.

Suppose now that the given chain transformations $\alpha$ and $\beta$ are chain homotopic only in dimensions not greater than $q-1$; that is, that the homomorphisms (3.3) and the relations (3.4) are given only for $i=0, \cdots, q-1$. In this event, using (3.4) for $i=q-1$, one has

$$
\begin{aligned}
\partial\left[\beta_{q}-\alpha_{q}-D_{q-1} \partial\right] & =\beta_{q-1} \partial-\alpha_{q-1} \partial-\partial D_{q-1} \partial \\
& =\alpha_{q-1} \partial+\partial D_{q-1} \partial+D_{q-2} \partial \partial-\alpha_{q-1} \partial-\partial D_{q-1} \partial=0 .
\end{aligned}
$$

Hence

$$
E_{q}=\beta_{q}-\alpha_{q}-D_{q-1} \partial
$$

is an operator homomorphism

$$
E_{q}: C_{q}\left(K_{1}\right) \rightarrow Z_{q}\left(K_{2}\right)
$$

into the group $Z_{q}\left(K_{2}\right)$ of integral $q$-cycles of $K_{2}$, such that

$$
\beta_{q}=\alpha_{q}+E_{q}+D_{q-1} \partial \text {. }
$$


Conversely, given $\alpha: K_{1}^{q} \rightarrow K_{2}^{q}$ and $D_{0}, \cdots, D_{q-1}, E_{q}$ as in (3.3) and (3.6), the definitions (3.4) and (3.7) yield an equivariant chain transformation $\beta: K_{1}^{q}$ $\rightarrow K_{2}^{q}$ chain homotopic to $\alpha$ in dimensions not greater than $q-1$.

The behavior of homotopies for products is indicated in the following lemma, where the chain transformations given may be defined only through the indicated dimensions, or for any higher dimensional skeletons.

LEMMA 3.1. Given equivariant chain transformations

$$
\mu: K_{0}^{q} \rightarrow K_{1}^{q} ; \quad \alpha, \beta: K_{1}^{q} \rightarrow K_{2}^{q} ; \quad \nu: K_{2}^{q+1} \rightarrow K_{3}^{q+1} ;
$$

the homotopy $\alpha \simeq \beta$ (dim $\leqq q)$ implies the homotopies $\alpha \mu \simeq \beta \mu(\operatorname{dim} \leqq q)$ and $\nu \alpha \simeq \nu \beta(\operatorname{dim} \leqq q)$.

Proof. If $D: \alpha \simeq \beta \quad(\operatorname{dim} \leqq q)$, then clearly $D \mu: \alpha \mu \simeq \beta \mu \quad(\operatorname{dim} \leqq q)$ and $\nu D: \nu \alpha \simeq \nu \beta(\operatorname{dim} \leqq q)$. Note that in the second case one must assume that $\nu$ acts as an equivariant chain transformation on the $(q+1)$-dimensional skeletons, in order to define the product $(\nu D)_{q}=\nu_{q+1} D_{q}: C_{q}\left(K_{1}\right) \rightarrow C_{q+1}\left(K_{3}\right)$.

For $q=\infty$, the homotopy $D: \alpha \simeq \beta$ becomes a homotopy in all dimensions; that is, (3.3) and (3.4) must hold for all integers $i$. Lemma 3.1 is still valid in this case.

4. Obstructions of chain transformations. If an equivariant chain transformation $\alpha: K_{1}^{q} \rightarrow K_{2}^{q}$ cannot be extended to the $(q+1)$-skeletons, one may introduce a suitable measure of the "obstruction" to this extension. By the basic condition (3.1), $\partial \alpha_{q} \partial c=\alpha_{q-1} \partial \partial c=0$ for any $(q+1)$-chain $c$ of $K_{1}$. Since $\alpha_{q} \partial c$ is thus a $q$-cycle in $K_{2}$, the mapping $\eta: Z_{q}\left(K_{2}\right) \rightarrow H_{q}\left(K_{2}\right)$ of (1.2) may thus be used to define an operator homomorphism

$$
l_{\alpha}^{q+1}=\eta \alpha_{q} \partial: C_{q+1}\left(K_{1}\right) \rightarrow H_{q}\left(K_{2}\right) .
$$

Alternatively, we may regard $l_{\alpha}^{q+1}$ as an equivariant $(q+1)$-cochain (cf. $\$ 1$ ) of $K_{1}$, with coefficients in $H_{q}\left(K_{2}\right)\left({ }^{3}\right)$.

By definition of the coboundary operator

$$
\delta l^{q+1}=l^{q+1} \partial=\eta \alpha_{q} \partial \partial=0 .
$$

Hence $l_{\alpha}^{a+1}$ is an equivariant cocycle.

LEMma 4.1. Each equivariant chain transformation $\alpha: K_{1}^{q} \rightarrow K_{2}^{q}$ determines as in (4.1) an obstruction $l_{\alpha}^{a+1}$ which is in $Z_{e}^{q+1}\left(K_{1}, H_{q}\left(K_{2}\right)\right)$. If $\alpha$ has an equivariant extension $\alpha: K_{1}^{q+1} \rightarrow K_{2}^{q+1}$ to the $(q+1)$-skeletons, the obstruction $l_{\alpha}^{q+1}=0$. Conversely, if $W$ operates freely on $C_{q+1}\left(K_{1}\right)$ and if the obstruction $l_{\alpha}^{++1}=0$, $\alpha$ has an extension $\alpha: K_{1}^{q+1} \rightarrow K_{2}^{\alpha+1}$.

Proof. Given an extension $\alpha_{q+1}$, one has $l^{q+1}=\eta \alpha_{g} \partial=\eta \partial \alpha_{q+1}=0$, since $\eta$

(3) If $q=0$, the obstruction $l_{\alpha}^{1}$ is defined and all its subsequent properties hold provided the reduced group $\bar{H}_{0}\left(K_{2}\right)$ is employed as coefficient group. 
annihilates the boundaries. Conversely, if $l^{q+1}=0, \alpha_{q} \partial$ is an operator homomorphism of $C_{q+1}\left(K_{1}\right)$ to $B_{q}\left(K_{2}\right)$, and by Lemma 2.1 can be lifted to a homomorphism $\alpha_{q+1}: C_{q+1}\left(K_{1}\right) \rightarrow C_{q+1}\left(K_{2}\right)$ such that $\partial \alpha_{q+1}=\alpha_{q} \partial$. This gives the required extension.

LEMMA 4.2. Two equivariant chain transformations $\alpha$ and $\beta: K_{1}^{q} \rightarrow K_{2}^{q}$ with $\alpha \simeq \beta(\operatorname{dim} \leqq q-1)$ determine cohomologous obstructions $l_{\alpha}^{a+1}$ and $l_{\beta}^{q+1}$. Conversely, if $\alpha: K_{1}^{q} \rightarrow K_{2}^{q}$ has an obstruction $l^{q+1}$ cohomologous to an equivariant cocycle $m^{q+1} \in Z_{e}^{q+1}\left(K_{1}, H_{q}\left(K_{2}\right)\right)$ and if $W$ operates freely on $C_{q}\left(K_{1}\right)$ there exists an equivariant chain transformation $\beta: K_{1}^{q} \rightarrow K_{2}^{q}$ with obstruction $m^{q+1}$ and with $\alpha_{i}=\beta_{i}$, for $i \leqq q-1$.

Proof. Given $D: \alpha \simeq \beta(\operatorname{dim} \leqq q-1),(3.7)$ gives

$$
\beta_{q} \partial=\alpha_{q} \partial+E_{q} \partial+D_{q-1} \partial \partial=\alpha_{q} \partial+E_{q} \partial .
$$

If we define the equivariant $q$-cochain $h^{q} \in C_{e}^{q}\left(K_{1}, H_{q}\left(K_{2}\right)\right)$ as $h^{q}=\eta E_{q}$, then $\delta h^{q}=\eta E_{q} \partial$, and the above equation yields $\eta \beta_{q} \partial=\eta \alpha_{q} \partial+\delta h^{q}$, or $l_{\beta}=l_{\alpha}$ $+\delta h^{q}$; thus the obstructions of $\beta$ and $\alpha$ are cohomologous. Conversely, given $m^{q+1}=l_{\alpha}+\delta h^{q}$, where $h^{q}: C_{q}\left(K_{1}\right) \rightarrow H_{q}\left(K_{2}\right)$, use Lemma 2.1 to lift $h^{q}$ to an operator homomorphism $E_{q}: C_{q}\left(K_{1}\right) \rightarrow Z_{q}\left(K_{2}\right)$ such that $\eta E_{q}=h^{q}$. Setting $\beta_{i}=\alpha_{i}$ for $i<q$ and $\beta_{q}=\alpha_{q}+E_{q}$ then yields a chain transformation $\beta: K_{1}^{q} \rightarrow K_{2}^{q}$ with $\alpha=\beta(\operatorname{dim} \leqq q-1)$ and with obstruction

$$
l_{\beta}=\eta \beta_{q} \partial=\eta \alpha_{q} \partial+\eta E_{q} \partial=l_{\alpha}+h^{q} \partial=l_{\alpha}+\delta h^{q}=m^{q+1},
$$

as desired.

CoRollary 4.3. Let $W$ operate freely on $C_{q}\left(K_{1}\right)$. Then the obstruction $l_{\alpha}^{q+1}$ of an equivariant chain transformation $\alpha: K_{1}^{q} \rightarrow K_{2}^{q}$ is an (equivariant) coboundary if and only if there is an equivariant chain transformation $\beta: K_{1}^{q+1}$ $\rightarrow K_{2}^{q+1}$ with $\beta_{i}=\alpha_{i}$ for $0 \leqq i \leqq q-1$.

The concept of the obstruction of a chain transformation is the combinatorial equivalent of the well known notion of the obstruction of a continuous mapping of one space into another (cf., for example, Eilenberg [18, p. 72 $)$. It would also be possible to construct an obstruction of a chain homotopy $D: \alpha \simeq \beta$ (dim $\leqq q-1)$; in fact, the homomorphism $E_{q}$ of (3.4) induces a homomorphism $\eta E_{q}: C_{q}\left(K_{1}\right) \rightarrow H_{q}\left(K_{2}\right)$, which can thus be regarded as a cochain in $C^{q}\left(K_{1}, H_{q}\left(K_{2}\right)\right)$. This cochain acts as the obstruction of $D$, in the sense that analogues of Lemmas 4.1 and 4.2 and of Corollary 4.3 can be proved.

5. The construction of chain transformations.

THEOREM 5.1. If $K_{1}, K_{2}$ are augmentable complexes with operators $W$ such that

(i) $K_{2}$ is acyclic in dimensions less than $q$, 
(ii) $W$ operates freely on the groups $C_{i}\left(K_{1}\right)$, for $0 \leqq i \leqq q$ ( $q$ is a positive integer or $\infty)$,

then there exists an equivariant chain transformation $\alpha: K_{1}^{q} \rightarrow K_{2}^{q}$, and any two such transformations are chain homotopic in dimensions not greater than $q-1$. Hence the cohomology class $1^{q+1}=\left\{l_{\alpha}^{a+1}\right\}$ of the obstruction of an $\alpha$ is independent of the choice of $\alpha$, and depends only on $K_{1}$ and $K_{2}$. If $W$ operates freely on $C_{q+1}\left(K_{1}\right)$, this class is zero if and only if there exists an equivariant $\beta: K_{1}^{q+1} \rightarrow K_{2}^{q+1}$.

Proof. We first construct the homomorphisms $\alpha_{i}$. To obtain $\alpha_{0}$, use the given operator homomorphisms $J: C_{0}\left(K_{1}\right) \rightarrow$ integers and $J: C_{0}\left(K_{2}\right) \rightarrow$ integers. The second is onto, by assumption (ii) of $\$ 1$; hence the first, by Lemma 2.1 , can be lifted to an operator homomorphism $\alpha_{0}: C_{0}\left(K_{1}\right) \rightarrow C_{0}\left(K_{2}\right)$ such that $J \alpha_{0}=J$; that is, such that $\alpha_{0}$ is augmentable. Since $J \alpha_{0} \partial=J \partial=0$ the homomorphism $\alpha_{0} \partial$ maps $C_{1}\left(K_{1}\right)$ into reduced cycles of $K_{2}$ and hence, by assumption (i), into boundaries $B_{0}\left(K_{2}\right)$. Because of assumption (ii) one can then by Lemma 2.1 lift $\alpha_{0} \partial$ to an equivariant homomorphism $\alpha_{1}: C_{1}\left(K_{1}\right) \rightarrow C_{1}\left(K_{2}\right)$ such that $\partial \alpha_{1}=\alpha_{0} \partial$.

By induction assume now that an equivariant chain transformation $\alpha: K_{1}^{t-1} \rightarrow K_{2}^{t-1}$ has been constructed for some $t, 1<t \leqq q$. Since $H_{t-1}\left(K_{2}\right)=0$, by assumption (i), the obstruction of $\alpha$ is a cocycle in the group $Z_{e}^{t}\left(K_{1}, 0\right)=0$, hence is zero. Therefore Lemma 4.1 provides an extension of $\alpha$ to the $t$-dimensional skeletons. This process continues up to $t=q$, to give $\alpha: K_{1}^{q} \rightarrow K_{2}^{q}$, as asserted in the theorem.

Consider next two transformations $\alpha, \beta: K_{1}^{q} \rightarrow K_{2}^{q}$. Since $J \beta_{0}-J \alpha_{0}=J-J$ $=0$, the homomorphism $\beta_{0}-\alpha_{0}$ maps $C_{0}\left(K_{1}\right)$ into reduced cycles of $K_{2}$ and hence, by assumption (i), into boundaries $B_{0}\left(K_{2}\right)$. Because of assumption (ii) one can then by Lemma 2.1 lift $\beta_{0}-\alpha_{0}$ to an operator homomorphism $D_{0}: C_{0}\left(K_{1}\right) \rightarrow C_{1}\left(K_{2}\right)$ such that $\beta_{0}-\alpha_{0}=\partial D_{0}$. Thus $D_{0}: \alpha \simeq \beta$ (dim $\left.\leqq 0\right)$. Again suppose by induction that $D: \alpha \simeq \beta(\operatorname{dim} \leqq t-1)$, for some $t$ with $1 \leqq t<q$, where the $D_{i}$ are homomorphisms $D_{i}: C_{i}\left(K_{1}\right) \rightarrow C_{i+1}\left(K_{2}\right)$ for $0 \leqq i<t$. Then $E_{t}$, defined as in (3.4) as $E_{t}=\beta_{t}-\alpha_{t}-D_{t-1} \partial$, is a homomorphism of $C_{t}\left(K_{1}\right)$ into $Z_{t}\left(K_{2}\right)$. By assumption (ii) $Z_{t}\left(K_{2}\right)=B_{t}\left(K_{2}\right)$, while $\partial$ is a homomorphism of $C_{t+1}\left(K_{2}\right)$ onto $B_{t}\left(K_{2}\right)$. By Lemma $2.1, E_{t}$ can thus be lifted to an operator homomorphism $D_{t}: C_{t}\left(K_{1}\right) \rightarrow C_{t+1}\left(K_{2}\right)$ such that $E_{t}=\beta_{t}-\alpha_{t}-D_{t-1} \partial=\partial D_{t}$. This conclusion gives the homotopy $D: \alpha \simeq \beta$ (dim $\leqq t)$, which completes the induction.

The remaining conclusions of the theorem follow by Corollary 4.3.

In the case $q=\infty$, the obstruction does not occur, so that the theorem has the following simpler form:

COROLlARY 5.2. If $K_{1}, K_{2}$ are augmentable complexes with operators $W$ such that

(i) $K_{2}$ is acyclic in all dimensions, 
(ii) $W$ operates freely on $K_{1}$,

there exists an equivariant chain transformation $\alpha: K_{1} \rightarrow K_{2}$, and any two such transformations are chain homotopic.

CoRollary 5.3. If $K_{1}$ and $K_{2}$ are augmentable complexes with operators $W$ such that

(i) $K_{1}$ and $K_{2}$ are acyclic in all dimensions,

(ii) $W$ operates freely on $K_{1}$ and $K_{2}$, then there exist equivariant chain transformations

$$
\alpha: K_{1} \rightarrow K_{2}, \quad \beta: K_{2} \rightarrow K_{1}
$$

such that $\beta \alpha$ and $\alpha \beta$ are (equivariantly) chain homotopic to the identity maps of $K_{1}$ and $K_{2}$ respectively. The transformations $\alpha$ and $\beta$ are unique up to chain homotopy.

Note that all these theorems apply in the case when $W$ consists of the identity alone. The case of ordinary closure finite abstract complexes, with ordinary chain transformations and chain homotopies, is thus included. For such complexes, the assumption (ii) of Theorem 5.1, as to free operators, holds automatically.

6. The characteristic cocycle of a complex with operators. For any group $W$ one may construct a "standard" associated complex $K_{W}$ with operators $W$. The $q$-cells of $K_{W}$ are the ordered $(q+1)$-tuples $\left(w_{0}, \cdots, w_{q}\right)$ of elements of $W$, with possible repetitions, and the boundary is defined as

$$
\partial\left(w_{0}, \cdots, w_{q}\right)=\sum_{i=0}^{q}(-1)^{i}\left(w_{0}, \cdots, \widehat{w}_{i}, \cdots, w_{q}\right), \quad q>0,
$$

where the circumflex over $w_{i}$ indicates that this term is to be omitted. This gives a closure finite augmentable complex, which has operators $w \in W$ defined by $w\left(w_{0}, \cdots, w_{q}\right)=\left(w_{0}, \cdots, w_{q}\right)[19, \S 12]$. An equivariant $q$-cochain of $K_{W}$ with coefficients in a group $G$ with $W$ as left operators is therefore a function $f$ of $q+1$ variables in $W$, with values in $G$ and subject to the condition $w f\left(w_{0}, \cdots, w_{q}\right)=f\left(w_{0}, \cdots, w_{q}\right)$. These equivariant cochains of $K_{W}$ will be called the cochains of $W$ over $G$, and the group of these cochains will be denoted by $C^{q}(W, G)$; similarly, $Z^{q}(W, G)$ and $B^{q}(W, G)$ will denote the groups of equivariant $q$-cocycles and of equivariant $q$-coboundaries of $K_{W}$. The group $H^{q}(W, G)=Z^{q}(W, G) / B^{q}(W, G)$ is called the $q$ th cohomology group of the group $W$.

The complex $K_{W}$ is acyclic in all dimensions $[19, \S 12]$, and its chain groups have free operators $W$, for the cells $\left(1, w_{1}, \cdots, w_{q}\right)$ constitute a $W$-base. Thus $K_{W}$ may serve as the complex $K_{1}$ of Theorem 5.1 , to give at once the following result.

THEOREM 6.1. If the augmentable complex $K$ with operators $W$ is acyclic in 
dimensions less than $q$, there is an equivariant chain transformation $\alpha: K_{W}^{q} \rightarrow K^{q}$. If $l^{q+1} \in Z^{q+1}\left(W, H_{q}(K)\right)$ denotes the obstruction of $\alpha$, a change in the choice of $\alpha$ alters $l^{a+1}$ by an equivariant coboundary, and any specified such alteration can be effected by a suitable choice of $\alpha$. The complex $K$ thus determines uniquely a characteristic cohomology class $1^{q+1}(K) \in H^{q+1}\left(W, H_{q}(K)\right)$.

Let $K_{1}, K_{2}$ be two augmentable complexes with operators $W$, both acyclic in dimensions less than $q$, while $\tau: K_{1} \rightarrow K_{2}$ is an augmentable equivariant chain transformation. Then $\tau$ induces an operator homomorphism $\tau: H_{q}\left(K_{1}\right)$ $\rightarrow H_{q}\left(K_{2}\right)$, and hence a homomorphism

$$
H^{q+1}\left(W, H_{q}\left(K_{1}\right)\right) \rightarrow H^{q+1}\left(W, H_{q}\left(K_{2}\right)\right) .
$$

The following theorem gives the action of this homomorphism on the characteristic classes.

TheOREM 6.2. An augmentable equivariant chain transformation $\tau: K_{1} \rightarrow K_{2}$ maps the characteristic cohomology class $1^{q+1}\left(K_{1}\right)$ under $(6.1)$ on the characteristic cohomology class $1^{a+1}\left(K_{2}\right)$.

Proof. If $\alpha: K_{W}^{q} \rightarrow K_{1}^{q}$ has obstruction $l_{1}$, then $\tau \alpha: K_{W}^{q} \rightarrow K_{2}^{q}$ is also a chain transformation, with an obstruction $l_{2}$ which is given, for each $c \in C_{q+1}\left(K_{W}\right)$, as

$$
l_{2}(c)=\eta \tau \alpha \partial c=\tau \eta \alpha \partial c=\tau l_{1} c .
$$

This gives $l_{2}=\tau l_{1}$, as asserted.

The characteristic cohomology class of $K$ may be obtained not from the equivariant chain transformation $\alpha$, but from a suitable sequence of cocycles. Indeed $\alpha: K_{W}^{q} \rightarrow K^{q}$ is simply a sequence of equivariant homomorphisms $\alpha_{i}: C_{i}\left(K_{W}\right) \rightarrow C_{i}(K)$, and each homomorphism $\alpha_{i}$ may be regarded as a cochain $f^{i} \in C^{i}\left(W, C_{i}(K)\right)$. Any such cochain $f^{i}$ has a coboundary $\delta f^{i}$ in $C^{i+1}\left(W, C_{i}(K)\right)$ and also a "boundary" defined for any $i$-cell of $K_{W}$ as

$$
\left(\partial f^{i}\right)\left(w_{1}, \cdots, w_{i}\right)=\partial\left[f^{i}\left(w_{0}, \cdots, w_{i}\right)\right] ;
$$

hence $\partial f^{i} \in C^{i}\left(W, C_{i-1}(K)\right)$. The condition $\alpha_{i} \partial=\partial \alpha_{i+1}$ that $\alpha$ be a chain transformation now becomes the condition $\delta f^{i}=\partial f^{i+1}$, while the condition that $\alpha$ is augmentable becomes the requirement that $f^{0}(1)$ have the sum of its coefficients 1 . Theorem 6.1 may thus be translated as follows:

THEOREM 6.3. If $K$ is an augmentable complex with operators $W$, acyclic in dimensions less than $q$, there exists a sequence of cochains $f^{i} \in C^{i}\left(W, C_{i}(K)\right)$ for $i=0, \cdots, q$ with

$$
J f^{0}(1)=1, \quad \delta f^{i}=\partial f^{i+1}, \quad i=0, \cdots, q-1 .
$$

For any such sequence, $\delta f^{q}$ has values in $Z_{q}(K)$, and the homomorphism $\eta: Z_{q}(K)$ $\rightarrow H_{q}(K)$ yields a cocycle $l^{q+1}=\eta \delta f^{q} \in Z^{q+1}\left(W, H_{q}(K)\right)$. The cohomology class of 
$l^{q+1}$ is independent of the choice of the sequence $f^{i}$ subject to the indicated conditions, and is the characteristic cohomology class of $K$.

This result is sometimes convenient as a method of calculating $1^{q+1}(K)$; clearly the $f^{i}$ 's used may be either homogeneous or nonhomogeneous cochains of the group $W$, cf. $[7, \S 2]$, and $\$ 26$ below.

7. Complexes with equal characteristic cocycles.

THEOREM 7.1. Let $K, K^{\prime}$ be augmentable complexes with free operators $W$ which are acyclic in dimensions less than $q$ and which have the same qth homology group $H$ and the same characteristic cohomology class $1 \in H^{q+1}(W, H)$. Then there exist equivariant chain transformations

$$
\lambda: K^{q+1} \rightarrow K^{\prime q+1}, \quad \lambda^{\prime}: K^{\prime q+1} \rightarrow K^{q+1},
$$

which induce the identity on the common qth homology group $H$, such that $\lambda \lambda \simeq I$ and $\lambda \lambda^{\prime} \simeq I^{\prime}(\operatorname{dim} \leqq q)$, where $I$ and $I^{\prime}$ are identity chain transformations $K \rightarrow K$ and $K^{\prime} \rightarrow K^{\prime}$.

The conclusion states essentially that $K, K^{\prime}$ have the same "chain homotopy type" in dimensions not greater than $q$, in a sense parallel to the usual (topological) homotopy type of spaces. The assumption that $K, K^{\prime}$ have the same $q$ th homology group $H$ means of course that there are homomorphisms of the groups of cycles

$$
\eta: Z_{q}(K) \rightarrow H, \quad \eta^{\prime}: Z_{q}\left(K^{\prime}\right) \rightarrow H
$$

onto the same $H$, with the groups $B_{q}(K)$ and $B_{q}\left(K^{\prime}\right)$ of boundaries as the respective kernels. The assumption that $K$ and $K^{\prime}$ have the same characteristic cohomology class means, in accordance with the definition of this class, that we may choose any cocycle $l$ in the given class 1 and equivariant transformations

$$
\alpha: K_{W}^{q} \rightarrow K^{q}, \quad \alpha^{\prime}: K_{W}^{q} \rightarrow K^{\prime q}
$$

with obstruction $l$. Then, as in the definition (4.1)

$$
\eta \alpha_{q} \partial=l, \quad \eta^{\prime} \alpha_{q}^{\prime} \partial=l
$$

Proof. Since $K_{W}$ is acyclic and $W$ operates freely on $K$ and $K^{\prime}$, Theorem 5.1 yields equivariant chain transformations

$$
\gamma: K^{q+1} \rightarrow K_{W}^{q+1}, \quad \gamma^{\prime}: K^{\prime q+1} \rightarrow K_{W}^{q+1}
$$

and homotopies

$$
D: \alpha \gamma \simeq I, \quad D^{\prime}: \alpha^{\prime} \gamma^{\prime} \simeq I^{\prime} \quad(\operatorname{dim} \leqq q-1) .
$$

As in (3.5) and (3.6), $E_{q}=I_{q}-\alpha_{q} \gamma_{q}-D_{q-1} \partial$ is an operator homomorphism

$$
E_{q}: C_{q}(K) \rightarrow Z_{q}(K), \quad E_{q}^{\prime}: C_{q}\left(K^{\prime}\right) \rightarrow Z_{q}\left(K^{\prime}\right) .
$$


Then $\eta E_{q}$ maps $C_{q}(K)$ into $H$, while $\eta^{\prime}: Z_{q}\left(K^{\prime}\right) \rightarrow H$ is onto, so that $\eta E_{q}$ can be lifted to a homomorphism $F_{q}: C_{q}(K) \rightarrow Z_{q}\left(K^{\prime}\right)$ such that

$$
\eta^{\prime} F_{q}=\eta E_{q}, \quad \eta F_{q}^{\prime}=\eta^{\prime} E_{q}^{\prime}
$$

Define

$$
\lambda: K^{q} \rightarrow K^{\prime q}, \quad \lambda^{\prime}: K^{\prime q} \rightarrow K^{q}
$$

by setting

$$
\begin{array}{lll}
\lambda_{i}=\alpha_{i}^{\prime} \gamma_{i}, & \lambda_{i}^{\prime}=\alpha_{i} \gamma_{i}^{\prime}, & 0 \leqq i<q, \\
\lambda_{q}=\alpha_{q}^{\prime} \gamma_{q}+F_{q}, & \lambda_{q}^{\prime}=\alpha_{q} \gamma_{q}^{\prime}+F_{q .}^{\prime} &
\end{array}
$$

Since $\partial F_{q}=0, \lambda$ and $\lambda^{\prime}$ are chain transformations. The obstruction of $\lambda$ is, by (7.3),

$$
\begin{aligned}
l_{\lambda} & =\eta^{\prime} \lambda_{q} \partial=\eta^{\prime} \alpha_{q}^{\prime} \gamma_{q} \partial+\eta^{\prime} F_{q} \partial \\
& =\eta^{\prime} \alpha_{q}^{\prime} \partial \gamma_{q+1}+\eta E_{q} \partial \\
& =l \gamma_{q+1}+\eta \partial-\eta \alpha_{q} \gamma_{q} \partial-\eta D_{q-1} \partial \partial \\
& =l \gamma_{q+1}-\eta \alpha_{q} \partial \gamma_{q+1}=l \gamma_{q+1}-l \gamma_{q+1}=0
\end{aligned}
$$

Similarly $l_{\lambda^{\prime}}=0$. Thus, by Lemma $4.1, \lambda, \lambda^{\prime}$ admit extensions

$$
\lambda: K^{q+1} \rightarrow K^{\prime q+1}, \quad \lambda^{\prime}: K^{\prime+1} \rightarrow K^{q+1} .
$$

We next show that the transformation $\lambda$ induces the identical map of $H_{q}(K)=H$ on $H_{q}\left(K^{\prime}\right)=H$; in other words, for any $z \in Z_{q}(K)$,

$$
\eta z=\eta \lambda_{q} z, \eta^{\prime} z^{\prime}=\eta \lambda_{q}^{\prime} z^{\prime} .
$$

For, $\gamma_{q} z$ is a cycle in the acyclic complex $K_{W}$, hence a boundary $\partial c$ in $K_{W}$, while the definitions of $\lambda_{q}$ in (7.7), of $F_{q}$ in (7.5), and of $E_{q}$ give

$$
\begin{aligned}
\eta^{\prime} \lambda_{q} z & =\eta^{\prime} \alpha_{q}^{\prime} \gamma_{q} z+\eta^{\prime} F_{q} z=\eta^{\prime} \alpha_{q}^{\prime} \partial c+\eta E_{q} z \\
& =l c+\eta z-\eta \alpha_{q} \gamma_{q} z-\eta D_{q-1} \partial z \\
& =l c+\eta z-\eta \alpha_{q} \partial c \\
& =l c+\eta z-l c=\eta z .
\end{aligned}
$$

Since $\gamma \alpha: K_{W}^{q} \rightarrow K_{W}^{q}$, while $K_{W}$ is acyclic with free operators $W$, there exist homotopies

$$
L: \gamma \alpha \simeq I_{W}, \quad L^{\prime}: \gamma^{\prime} \alpha^{\prime} \simeq I_{W} \quad(\operatorname{dim} \leqq q),
$$

where $I_{W}: K_{W} \rightarrow K_{W}$ is the identity. By Lemma 3.1, we thus have the following homotopies

$$
D: \alpha \gamma \simeq I
$$




$$
\begin{gathered}
\alpha L^{\prime} \gamma: \alpha \gamma^{\prime} \alpha^{\prime} \gamma \simeq \alpha \gamma, \\
\lambda^{\prime} D^{\prime} \alpha^{\prime} \gamma: \lambda^{\prime} \alpha^{\prime} \gamma^{\prime} \alpha^{\prime} \gamma \simeq \lambda^{\prime} \alpha^{\prime} \gamma, \\
-\lambda^{\prime} \alpha^{\prime} L^{\prime} \gamma: \lambda^{\prime} \alpha^{\prime} \gamma \simeq \lambda^{\prime} \alpha^{\prime} \gamma^{\prime} \alpha^{\prime} \gamma
\end{gathered}
$$

all valid in dimensions less than $q$. The combination

$$
M=D+\alpha L^{\prime} \gamma-\lambda^{\prime} \alpha^{\prime} L^{\prime} \gamma+\lambda^{\prime} D^{\prime} \alpha^{\prime} \gamma
$$

is thus a homotopy

$$
M=\alpha \gamma^{\prime} \alpha^{\prime} \gamma \simeq I
$$

$(\operatorname{dim}<q)$.

(Note: this statement would still be correct if the last two terms of $M$ were omitted, but the presence of these terms is essential for the subsequent argument.) By the definition of $\lambda$ this also gives a homotopy $M: \lambda \lambda \simeq I(\operatorname{dim}<q)$.

To prove that this latter homotopy can be extended, we consider its "obstruction"

$$
E_{q}^{*}=I_{q}-\lambda_{q}^{\prime} \lambda_{q}-M_{q-1} \partial
$$

defined as in (3.5) as an operator homomorphism $E_{q}^{*}: C_{q}(K) \rightarrow Z_{q}(K)$. To compute $E_{q}^{*}$ use the formulas

$$
\begin{aligned}
& L_{q-1}^{\prime} \partial=I_{W}-\gamma_{q}^{\prime} \alpha_{q}^{\prime}-\partial L_{q}^{\prime}, \\
& D_{q-1}^{\prime} \partial=I-\alpha_{q}^{\prime} \gamma_{q}^{\prime}-E_{q}^{\prime}, \quad D_{q-1} \partial=I-\alpha_{q} \gamma_{q}-E_{q},
\end{aligned}
$$

and the definition of $\lambda_{q}, \lambda_{q}^{\prime}$ in (7.7). Then

$$
\begin{aligned}
E_{q}^{*} & =I-\lambda_{q}^{\prime}\left(\alpha_{q}^{\prime} \gamma_{q}+F_{q}\right)-D_{q-1} \partial-\alpha_{q} L_{q-1}^{\prime} \partial \gamma_{q}-\lambda_{q}^{\prime} D_{q-1}^{\prime} \partial \alpha_{q}^{\prime} \gamma_{q}+\lambda_{q}^{\prime} \alpha_{q}^{\prime} L_{q-1}^{\prime} \partial \gamma_{q} \\
& =\left(E_{q}-\lambda_{q}^{\prime} F_{q}\right)+\left(\alpha_{q}-\lambda_{q}^{\prime} \alpha_{q}^{\prime}\right) \partial L_{q}^{\prime} \gamma_{q}-\left(F_{q}^{\prime}-\lambda_{q}^{\prime} E_{q}^{\prime}\right) \alpha_{q}^{\prime} \gamma_{q} .
\end{aligned}
$$

Consider each of the three terms in parentheses separately. For each $c \in C_{q}(K), E_{q} c$ and $F_{q} c$ are cycles, hence by (7.8) and (7.5),

$$
\begin{aligned}
& \eta\left(E_{q}-\lambda_{q}^{\prime} F_{q}\right)=\eta E_{q}-\eta^{\prime} F_{q}=0, \\
& \eta\left(F_{q}^{\prime}-\lambda_{q}^{\prime} E_{q}^{\prime}\right)=\eta F_{q}^{\prime}-\eta^{\prime} E_{q}^{\prime}=0 .
\end{aligned}
$$

Furthermore

$$
\eta\left(\alpha_{q}-\lambda_{q}^{\prime} \alpha_{q}^{\prime}\right) \partial=\eta \alpha_{q} \partial-\eta \lambda_{q}^{\prime} \alpha_{q}^{\prime} \partial=l-\eta^{\prime} \alpha_{q}^{\prime} \partial=l-l=0 .
$$

Hence $\eta E_{q}^{*}=0$, so that $E_{q}^{*}: C_{q}(K) \rightarrow B_{q}(K)$. Since $C_{q}(K)$ is $W$-free, $E_{q}^{*}$ may be lifted to a homomorphism $M_{q}: C_{q}(K) \rightarrow C_{q+1}(K)$ such that

$$
\partial M_{q}=E_{q}^{*}=I_{q}-\lambda_{q}^{\prime} \lambda_{q}-M_{q-1} \partial .
$$

This asserts that $M: \lambda^{\prime} \lambda \simeq I(\operatorname{dim} \leqq q)$, as desired.

8. The normalized complex $K_{W}^{*}$. The complex $K_{W}$ which is used in the 
definition of the characteristic cocycle and of the cohomology groups $H^{q}(W, G)$ of the group $W$ could be replaced by any other specific augmentable complex with free operators $W$ which is acyclic in all dimensions. Another such complex, the "normalized" complex $K_{W}^{*}$, can be constructed, using as $q$-cells the $(q+1)$-tuples $\left(w_{0}, w_{1}, \cdots, w_{q}\right)$ of elements $w_{i}$ in $W$ such that no two adjacent elements are equal $\left(w_{0} \neq w_{1}, \cdots, w_{q-1} \neq w_{q}\right)$. Operators of $W$ on these cells are defined as in $K_{W}$, and the boundary operator is

$$
\left(w_{0}, \cdots, w_{q}\right)=\sum_{i=0}^{q} *(-1)^{i}\left(w_{0}, \cdots, w_{i-1}, w_{i+1}, \cdots, w_{q}\right), \quad q \geqq 1,
$$

where the star on the summation indicates that all terms with adjacent arguments equal $\left(w_{i-1}=w_{i+1}\right)$ are to be omitted. One may show that $K_{W}^{*}$ is an augmentable complex with free operators $W$, and is acyclic in all dimensions. The natural chain transformation of $K_{W}$ onto $K_{W}^{*}$ represents the latter as a "quotient complex" of the former. The inverse map on cochains shows that an equivariant cochain $f$ in $K_{W}^{*}$ may be regarded as an equivariant cochain in $K_{W}$, subject to the additional "normalization" condition that $f\left(w_{0}, \cdots, w_{q}\right)$ $=0$ whenever two adjacent arguments are equal.

By using Corollary 5.2, it can be shown that this transformation provides an isomorphism between the equivariant cohomology groups of $K_{W}^{*}$ and $K_{W}$. This isomorphism gives the fact that the cohomology group $H^{q}(W, G)$ can be represented by normalized cochains. This fact was proved by direct means in $[7, \S 6]$, using a different "non-homogeneous" expression for the cochains (see $\$ 26$ below).

9. Spaces with operators. Hitherto the discussion has been purely algebraic. Now consider a topological space $X$ on which the group $W$ acts as a group of transformations $w: X \rightarrow X$. The total singular complex $S(X)$, which is composed of all the singular simplices of $X$ (with ordered vertices), is then an augmentable complex with $W$ as operators $[19, \S 8]$. The space $X$ is said to be acyclic in dimensions less than $q$ if the complex $S(X)$ is acyclic in the same dimensions, and under these circumstances the characteristic cohomology class

$$
l^{q+1}(X) \in H^{q+1}\left(W, H_{q}(X)\right)
$$

is defined.

Lemma 9.1. $W$ acts freely on the complex $S(X)$ if and only if none of the transformations $w: X \rightarrow X$ with $w \neq 1$ has a fixed point.

Proof. Suppose that $w \neq 1$ and $w x=x$ for some point $x \in X$. If we treat the point $x$ as a singular 0 -simplex in $X$ it follows that the homomorphism $w: C_{0}(X) \rightarrow C_{0}(X)$ has a fixed point. Hence the group $C_{0}(X)$ cannot have a $W$-base. 
Conversely, suppose that none of the transformations $w: X \rightarrow X$ has a fixed point for $w \neq 1$. One can then choose a set of "representative" points $\bar{x}$ (a fundamental domain for $X$ under $W$ ) in such fashion that every point $x$ can be written uniquely as $x=w \bar{x}$; every singular simplex $T$ in $X$ can then be written uniquely as $T=w \bar{T}$, where the leading vertex of $\bar{T}$ is one of the representative points $\bar{x}$. These simplices $\bar{T}$ form a $W$-base for the group $C_{i}(X)$ of $i$-chains of $S^{\prime}(X)$, thus proving that $W$ operates freely on $S(X)$.

The singular complex $S(X)$ is too large for actual computations. In order to be able to compute examples one usually assumes that the space $X$ is a simplicial polyhedron $P$ (finite or locally finite) and that $W$ operates on $P$ as a group of simplicial transformations. One then replaces the complex $S(P)$ by a "smaller" complex. One of those is the complex $K(P)[3, \S 8]$ in which, roughly speaking, every simplex of $P$ occurs with all possible orderings of its vertices. The other one is the still "smaller" complex $k(P)$ in which every simplex occurs once, with a selected orientation. For these complexes, lemmas similar to the one above hold.

LEMMA 9.2. $W$ acts freely on the complex $K(P)$ if and only if none of the transformations $w: P \rightarrow P$ with $w \neq 1$ leaves a vertex fixed.

Lемма 9.3. $W$ acts freely on the complex $k(P)$ if and only if none of the transformations $w: P \rightarrow P$ with $w \neq 1$ has a fixed point in $P$.

The proof of Lemma 9.2 is like that of Lemma 9.1, except that the argument is restricted to the vertices of $P$.

To prove Lemma 9.3, assume that $w \neq 1$ and that $w: P \rightarrow P$ has a fixed point. Then there is a simplex $s$ in $P$ such that $w s=s$. Let the dimension of $s$ be $i$, and let $c \neq 0$ be a chain carried by $s$. Then $w c= \pm c$ which shows that $C_{i}(k(P))$ does not have a $W$-base.

Conversely, assume that no $w: P \rightarrow P$ has a fixed point for $w \neq 1$. A simplex of $P$ will be called basic if its barycenter is a representative point. Then the elementary chains carried by the basic simplexes form $W$-bases for the groups $C_{i}(k(P))$.

These lemmas collectively show that the fact that $W$ operates freely on a complex $K$ essentially expresses the idea that $W$ operates on the corresponding space without fixed points.

If $P$ is acyclic in dimensions less than $q$, then we can define three characteristic cohomology classes

$$
l_{S}^{q+1}, \quad l_{K}^{q+1}, \quad I_{k}^{q+1},
$$

one for each of the complexes $S(P), K(P)$, and $k(P)$, The three complexes are compared by means of natural chain transformations $[19, \S 9]$,

$$
k(P) \stackrel{\boldsymbol{\alpha}}{\leftarrow} K(P) \stackrel{\boldsymbol{\beta}}{\rightarrow} S(P) .
$$

Both $\alpha$ and $\beta$ are equivariant and induce operator isomorphisms 


$$
H_{q}(k(P)) \stackrel{\alpha *}{\leftarrow} H_{q}(K(P)) \stackrel{\beta *}{\rightarrow} H_{q}(S(P)) .
$$

These in turn induce isomorphisms

$$
H^{q+1}\left(W, H^{q+1}(k(P))\right) \stackrel{\alpha^{*}}{\leftarrow} H^{q+1}\left(W, H^{q+1}(K(P))\right) \stackrel{\beta^{*}}{\rightarrow} H^{q+1}\left(W, H_{q}(S(P))\right) .
$$

By Theorem 6.2, the cohomology classes $I_{S}^{q+1}, I_{k}^{q+1}, I_{K}^{q+1}$ correspond to each other under these isomorphisms

$$
\alpha^{*} 1_{K}^{q+1}=1_{k}^{q+1}, \quad \beta^{*} 1_{K}^{q+1}=1_{S}^{q+1} .
$$

This shows that $I_{K}^{++1}$ and $I_{k}^{\alpha+1}$ are topological invariants of $P$ as a space and are independent of the particular simplicial decomposition of $P$. We shall write

$$
l^{q+1}(P) \in H^{q+1}\left(W, H_{q}(P)\right)
$$

for this invariant, without indicating which of the three definitions is used.

We consider subsequently the equivariant cohomology groups of $P$. By $[19, \S \S 8-10]$, the transformation $\beta: K(P) \rightarrow S(P)$ induces an isomorphism $H_{e}^{q}(S(P), G) \cong H_{e}^{q}(K(P), G)$. If, in addition, we assume that the operators are regular, in the sense of $[19, \S 10]$, it follows also that $\alpha$ induces an isomorphism $H_{e}^{q}(k(P), G) \cong H_{e}^{q}(K(P), G)$, so that under these circumstances the equivariant cohomology groups coincide.

For actual computations of $1^{q+1}(P)$ it is often more convenient to represent $P$ as an abstract complex $K_{1}$ that is not simplicial but can be subdivided into a simplicial complex $K_{2}$. In order for $K_{1}$ to be useful for computations it is necessary that the subdivision operator

$$
S d: K_{1} \rightarrow K_{2}
$$

induce isomorphisms

$$
H_{q}\left(K_{1}\right) \cong H_{q}\left(K_{2}\right) \text {. }
$$

If $K_{1}, K_{2}$, and $S d$ are invariant under the operators $W$, Theorem 6.2 shows that the characteristic cohomology class $1^{q+1}$, calculated from $K_{1}$, will correspond to the same invariant calculated from $K_{2}$.

\section{Chapter II. Computation of Equivariant groups}

10. Decomposition of the cohomology groups. There is a natural decompotion of the $q$ th equivariant cohomology group of a complex $K$ with operators. First let $K$ be any abstract closure finite complex (not necessarily augmentable, or with operators $W)$. Let $G$ be an abelian group and let $f \in Z^{q}(K, G)$ be a $q$-dimensional cocycle of $K$ over $G$. The fact that $f$ is a cochain means that $f$ is a homomorphism

$$
f: C_{g}(K) \rightarrow G
$$


The fact that $f$ is a cocycle means that $(\delta f)(c)=f(\partial c)=0$ for every $c \in C_{q+1}(K)$, or, equivalently, that

$$
f: B_{q}(K) \rightarrow 0 .
$$

Consequently, $f$ induces a homomorphism

$$
\theta_{f}: H_{q}(K) \rightarrow G,
$$

where $H_{q}(K)=Z_{q}(K) / B_{q}(K)$ is the $q$ th integral homology group of $K$. If $f$ is a coboundary $f=\delta h$, then for every $z \in Z_{q}(K)$, we have

$$
f(z)=\delta h(z)=h(\partial z)=0 ;
$$

hence $\theta_{f}=0$. Consequently, the correspondence $f \rightarrow \theta_{f}$ defines a homomorphism

$$
\theta: H^{q}(K, G) \rightarrow \operatorname{Hom}\left(H_{q}(K), G\right)
$$

of the $q$ th cohomology group of $K$ over $G$ into the group of all homomorphisms of $H_{q}(K)$ into $G$.

LEMMA 10.1. The homomorphism (10.1) is onto. If $H_{q-1}(K)=0$, then (10.1) is an isomorphism.

Proof. A proof of this lemma may be found in the literature; for the sake of completeness, however, we shall give a proof here. We observe first that, since $C_{q}(K) / Z_{q}(K) \cong B_{q-1}(K)$ and $B_{q-1}(K)$, as a subgroup of $C_{q-1}(K)$, is a free abelian group, it follows that $Z_{q}(K)$ is a direct summand of $C_{q}(K)$.

First take any $\alpha \in \operatorname{Hom}\left(H_{q}(K), G\right)$. This defines a homomorphism $\rho: Z_{q}(K) \rightarrow G$ with $B_{q}(K) \rightarrow 0$. Since $Z_{q}$ is a direct summand of $C_{q}, \rho$ may be extended to a homomorphism $f: C_{q}(K) \rightarrow G$. This $f$ is a cocycle, and $\theta_{f}=\rho$.

Assume now that $H_{q-1}(K)=0$, and let $f$ be a $q$-cocycle with $\theta_{f}=0$. Then

$$
f: C_{q}(K) \rightarrow G, \quad f: Z_{q}(K) \rightarrow 0 .
$$

Thus $f$ induces a homomorphism $h_{0}: B_{q-1}(K) \rightarrow G$ defined by $f(c)=h_{0}(\partial c)$ for every $q$-chain $c$. Since $H_{q-1}(K)=0$, we have $B_{q-1}=Z_{q-1}$, and since $Z_{q-1}$ is a direct summand of $C_{q-1}$, there is an extension $h: C_{q-1}(K) \rightarrow G$ of $h_{0}$. Then $h$ is $(q-1)$-cochain, and $\delta h=f$, as asserted in the lemma.

Let us now assume that the group $W$ operates both on $K$ and on $G$ (on the left). Then $W$ acts also as a group of (left) operators on $H_{q}(K)$, and in the group $\operatorname{Hom}\left(H_{q}(K), G\right)$ we may distinguish the subgroup Ophom $\left(H_{q}(K), G\right)$ of operator homomorphisms. If $f \in Z^{q}(K, G)$, then $\theta_{f}$ is an operator homomorphism if and only if

$$
f(w z)=w f(z) \quad \text { for } w \in W, z \in Z_{q}(K) .
$$

In particular, if $f$ is an equivariant cocycle, then $\theta_{f}$ is an operator homomorphism. This leads to a homomorphism

$$
\theta: H_{e}^{q}(K, G) \rightarrow \text { Ophom }\left(H_{q}(K), G\right) .
$$


Each equivariant cocycle is also an ordinary cocycle and determines an ordinary cohomology class; as in $[19, \S 4]$ this correspondence yields a homomorphism

$$
\phi: H_{e}^{q}(K, G) \rightarrow H^{q}(K, G)
$$

into the ordinary cohomology group of $K$. The kernel $N_{e}^{q}(K, G)$ of this homomorphism consists of the cohomology classes of those equivariant cocycles $f$ which have the form $f=\delta h$ for some (not necessarily equivariant) cochain $h$ of $C^{q}(K, G)$. The homomorphism $\theta$ of (10.2) may be obtained by combining (10.1) with the homomorphism $\phi$ and subsequently restricting the range. Hence $N_{e}^{q}$ is contained in the kernel of $\theta$ in (10.2).

Conversely, suppose $H_{q-1}(K)=0$. If $\theta_{f}=0$, Lemma 10.1 shows that $f=\delta h$ is an ordinary coboundary, hence lies in $N_{e}^{q}$. Thus in this case $N_{e}^{q}$ is the kernel of $\theta$. The result may be stated as follows.

THEOREM 10.2. Let $K$ be a complex with operators $W$, with $H_{q-1}(K)=0$. The homomorphism $\theta$ of (10.2) maps the equivariant cohomology group $H_{e}^{q}(K, G)$ into the group Ophom $\left(H_{q}(K), G\right)$, with kernel $N_{e}^{q}(K, G)$ equal to the kernel of the mapping $\phi$ of (10.3). Thus $\left(H_{e}^{q}, \theta\right)$ is a group extension of $N_{e}^{q}(K, G)$ by a subgroup of Ophom $\left(H_{q}(K), G\right)$.

The decomposition of $H_{e}^{q}$ by the homomorphism $\theta$ with kernel $N_{e}^{q}$ is transformed "naturally" by an equivariant chain transformation $\tau: K_{1}^{q+1}$ $\rightarrow K_{2}^{q+1}$. Specifically, $\tau$ induces in the usual fashion a homomorphism

$$
\tau^{*}: H_{e}^{q}\left(K_{2}, G\right) \rightarrow H_{e}^{q}\left(K_{1}, G\right) .
$$

The definition of $N$ as the kernel of $\phi$ above shows also that $\tau^{*}$ maps $N_{e}^{q}\left(K_{2}, G\right)$ into $N_{e}^{q}\left(K_{1}, G\right)$. This means that $N_{e}^{q}$ is a contravariant functor of $K$ in the sense of [20]. In the terminology there introduced, the homomorphism $\theta$ of (10.2) is "natural." Indeed, $\tau$ induces a mapping $\tau: H_{q}\left(K_{1}\right) \rightarrow H_{q}\left(K_{2}\right)$ on the homology groups, and thence a "reversed" mapping

$$
\tau: \text { Hom }\left(H_{q}\left(K_{2}\right), G\right) \rightarrow \operatorname{Hom}\left(H_{q}\left(K_{1}\right), G\right),
$$

defined as $\tau^{\#} \alpha_{2}=\alpha_{2} \tau$ for any $\alpha_{2} \in$ Hom $\left(H_{q}\left(K_{2}\right), G\right)$. The respective mappings $\theta_{2}, \theta_{1}$ of (10.2) map the first (second) groups of (10.4) into the corresponding group of (10.5). The assertion that $\theta$ is natural means that

$$
\tau \sharp \theta_{2}=\theta_{1} \tau^{*} \text {. }
$$

This may be proved by direct application of the relevant definitions.

If $\tau, \rho: K_{1}^{q+1} \rightarrow K_{2}^{q+1}$ are two equivariant chain transformations homotopic in dimensions not greater than $q$, a standard argument shows that the induced homomorphisms $\tau^{*}$ and $\rho^{*}$ on the $q$ th equivariant cohomology group are identical. The results of Theorem 7.1 on the transformations $\lambda$ and $\lambda^{\prime}$ constructed there may now be interpreted in terms of the cohomology groups, for 
$\lambda \lambda \simeq I(\operatorname{dim} \leqq q)$ and $\lambda \lambda^{\prime} \simeq I^{\prime}(\operatorname{dim} \leqq q)$ implies that $1=(\lambda \lambda)^{*}=\lambda^{*} \lambda^{\prime *}$ and similarly $\lambda^{\prime *} \lambda^{*}=1$, so that $\lambda^{*}$ and $\lambda^{\prime *}$ are (mutually inverse) isomorphisms on the cohomology groups.

THEOREM 10.3. If the augmentable complexes $K$ and $K^{\prime}$ with free operators $W$ satisfy the hypotheses of Theorem 7.1, there is an isomorphism

$$
\lambda^{*}: H_{e}^{q}\left(K^{\prime}, G\right) \leftrightarrow H_{e}^{q}(K, G)
$$

which maps $N_{e}^{q}\left(K^{\prime}, G\right)$ onto $N_{e}^{q}(K, G)$ and which induces the identity on the corresponding factor groups.

The last assertion means that the homomorphisms

$$
H_{e}^{q}(K, G) \stackrel{\theta^{\prime}}{\rightarrow} \text { Ophom }(H, G) \stackrel{\theta}{\leftarrow} H_{e}^{q}(K, G)
$$

are related by $\theta^{\prime}=\theta \lambda^{*}$.

This result shows that the $q$ th equivariant cohomology group of a complex $K$ with free operators $W$ acyclic in dimensions less than $q$ is determined by $W, H_{q}(K)$, the operation of $W$ on $H_{q}(K)$, and by the characteristic cohomology class $l^{a+1}(K)$. The algebraic form of this determination will now be obtained.

11. The main homomorphism. Let $K$ be an augmentable complex with operators $W$, acyclic in dimensions less than $q$; in accordance with Theorem 5.1 choose a chain transformation $\alpha: K_{W}^{q} \rightarrow K^{q}$, with obstruction $l \in Z^{q+1}\left(W, H_{q}(K)\right)$, given as $l=\eta \alpha_{q} \partial$, where $\eta$ is the natural mapping $Z_{q}(K) \rightarrow H_{q}(K)$. To analyze the equivariant cohomology group $H_{e}^{q}(K, G)$, where $G$ is a given abelian group with $W$ as (left) operators, we determine from each equivariant cocycle $f \in Z_{e}^{q}(K, G)$ the homomorphism $\theta_{f}$ of $\S 10$, as

$$
\theta_{f} \eta z_{g}=f\left(z_{q}\right) \text {, }
$$$$
\boldsymbol{z}_{q} \in Z_{q}(K),
$$

and a $q$-cochain $r_{f}$ of $K_{W}$, defined as

$$
r_{f}(c)=f\left(\alpha_{q} c\right),
$$$$
c \in C_{q}\left(K_{W}\right)
$$

We study the correspondence $\Gamma=\Gamma_{\alpha}$

$$
\Gamma f=\left(\theta_{f}, r_{f}\right), \quad f \in Z_{e}^{q}(K, G),
$$

carrying the cocycle $f$ into a certain pair of objects.

Since $f$ is equivariant,

$$
r_{f} w c=f \alpha_{q} w=f w \alpha_{q}=w f \alpha_{q} c=w r_{f},
$$

so that $r_{f}$ is an equivariant cochain of $K_{W}$. This, combined with (10.2), shows that

$$
\theta_{f} \in \text { Ophom }\left(H_{q}(K), G\right), r_{f} \in C^{q}(W, G) .
$$

If $d \in C_{q+1}\left(K_{W}\right)$, then 


$$
\left(\delta r_{f}\right)(d)=r_{f}(\partial d)=f\left(\alpha_{q} \partial d\right)=\theta_{f} \eta \alpha_{q} \partial d=\theta_{f} l d .
$$

Hence

$$
\delta r_{f}=\theta_{f} l
$$

Thus the correspondence $\Gamma$ carries $f$ into pairs satisfying the conditions (11.4) and (11.5). Since $\theta_{f+o}=\theta_{f}+\theta_{o}$ and $r_{f+o}=r_{f}+r_{o}$, this correspondence $\Gamma$ is a homomorphism. In particular, if $f \in B_{e}^{q}(K, G)$, then $f=\delta h$, for some $h \in C_{e}^{q-1}(K, G)$. In this case $\theta_{f}=0$ and

$$
r_{f}=f \alpha_{q}=(\delta h) \alpha_{q}=h \partial \alpha_{q}=h \alpha_{q-1} \partial=\delta\left(h \alpha_{q-1}\right) .
$$

But $s=h \alpha_{q-1}: C_{q-1}\left(K_{W}\right) \rightarrow G$ is a $q-1$ dimensional cochain of $K_{W}$, and is equivariant since $s w=h \alpha_{q-1} w=h w \alpha_{q-1}=w h \alpha_{q-1}=w s$. The equation above becomes $r_{f}=\delta s$; it asserts that $\Gamma$ carries coboundaries $f=\delta h$ into pairs $(0, \delta s)$, for $s \in C^{q-1}(W, G)$.

12. The group construction. The image of $\Gamma$ is a certain group of pairs depending on $W, G, H=H_{q}(K)$, and $l$. Such a group of pairs will now be constructed directly from any abelian groups $H$ and $G$ with $W$ as a group of left operators and any cocycle $l \in Z^{q+1}(W, H)$. Let $\rho$ be an operator homomorphism of $H$ into $G$ and $r$ a cochain of $C^{q}(W, G)$. Form the group $Z^{q}(W, H, l, G)$ as the group of those pairs $(\rho, r)$ in the direct sum

$$
\text { Ophom }(H, G)+C^{q}(W, G)
$$

which satisfy the condition

$$
\delta r\left(w_{0}, \cdots, w_{q+1}\right)=\rho\left[l\left(w_{0}, \cdots, w_{q+1}\right)\right], \quad w_{i} \in W ;
$$

the condition may be stated more briefly as

$$
\delta r=\rho l \text {. }
$$

Any pair $(0, r)$ with $\delta r=0$ satisfies this condition; hence each cocycle $r \in Z^{q}(W, G)$ may be identified with the element $(0, r) \in Z^{q}(W, H, l, G)$. Since $B^{q}(W, G)$ in turn is a subgroup of $Z^{q}(W, G)$, we may form the quotient group

$$
E^{q}(W, H, l, G)=Z^{q}(W, H, l, G) / B^{q}(W, G) .
$$

Then $H^{q}(W, G)=Z^{q}(W, G) / B^{q}(W, G)$ is a subgroup of $E^{q}$. The correspondence $(\rho, r) \rightarrow \rho$ is a homomorphism of $Z^{q}(W, H, l, G)$ into Ophom $(H, G)$ with kernel $Z^{q}(W, G)$, hence induces a homomorphism

$$
\chi: E^{q}(W, H, l, G) \rightarrow \text { Ophom }(H, G) .
$$

THEOREM 12.1. If $W$ operates on the abelian groups $H$ and $G$, each cocycle $l \in Z^{q+1}(W, H)$ determines an abelian group $E^{q}(W, H, l, G)$ and a homomorphism $\chi$ of $E^{q}$ into Ophom $(H, G)$. The kernel of $\chi$ is the group $H^{q}(W, G)$ regarded as a subgroup of $E^{q}$, and the image of $\chi$ is that subgroup $A(l)$ of $\operatorname{Ophom}(H, G)$ which consists of those operator homomorphisms $\rho: H \rightarrow G$ for which $\rho l$ is a 
coboundary: $\rho l \in B^{q+1}(W, G)$. Thus $\left(E^{q}, \chi\right)$ is an abelian group extension of $H^{q}(W, G)$ by $A(l)$. The subgroup $A(l)$ of Ophom and the extension in question are independent of the choice of the cocycle $l$ within its cohomology class in $H^{q+1}(W, H)$.

Proof. The kernel $\chi$ is clearly $H^{q}$. To study the image, take any pair $(\rho, r)$ in $Z^{p}(W, H, l, G)$; then, by $\left(12.2^{\prime}\right) \delta r=\rho l$, so that $\chi(\rho, r)=\rho$ has $\rho l$ a coboundary, so lies in $A(l)$ by definition. Conversely, any $\rho \in A(l)$ has $\rho l=\delta r$ for some cochain $r \in C^{q}(W, G)$, hence is the $\chi$-image of a pair $(\rho, r)$ of $Z^{q}(W, H, l, G)$.

Now let $l$ be changed to a cohomologous $l^{\prime}$, where

$$
l^{\prime}-l=\delta h
$$

for some $h \in C^{q}(W, H)$. Then for each $\rho \in \operatorname{Ophom}(H, G)$

$$
\rho l^{\prime}-\rho l=\rho \delta h=\delta \rho h,
$$

so that $\rho l$ is a coboundary, if and only if $\rho l^{\prime}$ is. Hence $A(l)=A\left(l^{\prime}\right)$.

Further, given $(\rho, r) \in Z^{q}\left(W, H, l^{\prime}, G\right)$, define

$$
\gamma_{h}(\rho, r)=(\rho, r-\rho h) .
$$

Then $\delta(r-\rho h)=\delta r-\delta \rho h=\delta r-\rho l^{\prime}+\rho l=\rho l$, and thus $\gamma_{h}(\rho, r) \in Z^{q}(W, H, l, G)$. It is easy to see that $\gamma_{h}$ establishes an isomorphism

$$
\gamma_{h}: Z^{q}\left(W, H, l^{\prime}, G\right) \cong Z^{q}(W, H, l, G) .
$$

Under this isomorphism each element of $Z^{q}(W, G)$ is mapped into itself. Hence $\gamma_{h}$ induces an isomorphism

$$
\gamma_{h}: E^{q}\left(W, H, l^{\prime}, G\right) \cong E^{q}(W, H, l, G)
$$

which leaves $H^{q}(W, G)$ pointwise fixed. Moreover

$$
\chi^{\prime}=\chi \gamma_{h}
$$

for $\chi \gamma_{h}(\rho, r)=\chi(\rho, r-\rho h)=\rho=\chi^{\prime}(\rho, r)$. This conclusion (12.4) means that the two extensions of $H^{q}(W, G)$ by $A(l)$ are equivalent (under the isomorphism $\gamma_{h}$ ) and thus concludes the proof of the theorem.

We may remark that the construction of the isomorphism $\gamma_{h}$ depended not only upon the choice of $l^{\prime}$ but also upon the choice of $h$. Hence if $l=l^{\prime}$, then $\delta h=0$, and $\gamma_{h}$ is an automorphism of $E^{q}(W, H, l, G)$ leaving $H^{q}(W, G)$ and $A(l)$ pointwise fixed. Thus the group $Z^{q}(W, H)$ acts as a group of operators on the group $E^{q}(W, H, l, G)$. Further, if $h \in B^{q}(W, H)$, then

$$
(\rho, r)-\gamma_{h}(\rho, r)=(\rho, r)-(\rho, r-\rho h)=(0, \rho h) \in B^{q}(W, G),
$$

and $\gamma_{h}$ is the identity map of $E$ onto itself. It follows that $H^{q}(W, H)$ acts on the group $E^{q}(W, H, l, G)$ as a group of operators which leave the subgroup $H^{q}(W, G)$ and the quotient group $A(l)$ pointwise fixed. 
Theorem 12.2. If $l$ is a coboundary, then $A(l)=$ Ophom $(H, G)$. Moreover the extension of Theorem 12.1 is then trivial, so that the group $E$ is a direct sum

$$
E^{q}(W, B, i, G) \cong H^{q}(W, G)+\text { Ophom }(H, G) .
$$

Proof. If $l$ is a coboundary, then $\rho l$ also is one for any $\rho \in \operatorname{Ophom}(H, G)$. Hence $A(l)=$ Ophom $(H, G)$. To prove the statement concerning the extension, we may, in view of Theorem 12.1, assume that $l=0$. Condition (12.2') then states that $r$ is a cocycle and therefore that

$$
Z^{q}(W, H, l, G)=\text { Ophom }(H, G)+Z^{q}(W, G),
$$

thus giving the conclusion.

We should remark that, even if $l \in Z^{q+1}(W, H)$ is not a coboundary, it may happen that $A(l)=$ Ophom $(H, G)$ for arithmetic reasons. If this takes place for every choice of $G$, then $l$ must be a coboundary, as is easily seen by choosing $G=H$ and $\rho: H \rightarrow G$ to be the identity.

13. Properties of the main homomorphism. Let $K$ be an augmentable complex with operators $W$, acyclic in dimensions less than $q$, and $\alpha: K_{W}^{q} \rightarrow K^{q}$ a chain transformation with obstruction $l$. The group $Z^{q}$ of the last section was so constructed that the mapping $\Gamma=\Gamma_{\alpha}$ of $\$ 11$ is a homomorphism

$$
\Gamma_{\alpha}: Z_{e}^{q}(K, G) \rightarrow Z^{q}\left(W, H_{q}(K), l, G\right) .
$$

LEMMA 13.1. The homomorphism $\Gamma_{\alpha}$ induces a homomorphism

$$
\Gamma_{\alpha}: H_{e}^{q}(K, G) \rightarrow E^{q}\left(W, H_{q}(K), l, G\right)
$$

of the equivariant cohomology group of $K$, and hence induces homomorphisms

$$
\begin{aligned}
\chi \Gamma_{\alpha}: H_{e}^{q}(K, G) & \rightarrow \text { Ophom }\left(H_{q}(K), G\right), \\
\Gamma_{\alpha}: N_{e}^{q}(K, G) & \rightarrow H^{q}(W, G) .
\end{aligned}
$$

The latter two homomorphisms are independent of the choice of $\alpha: K_{W}^{q} \rightarrow K^{q}$. Moreover, if $\phi, \theta$ are the homomorphisms

$$
\phi: H_{\bullet}^{q}(K, G) \rightarrow H^{q}(K, G), \quad \theta: H^{q}(K, G) \rightarrow \operatorname{Hom}\left(H_{q}(K), G\right)
$$

of (10.3) and (10.1), respectively, then

$$
\chi \Gamma_{\alpha}=\theta \phi .
$$

Proof. In $\S 11$, it was shown that the homomorphism $\Gamma_{\alpha}$ acting on cocycles carries $B_{e}^{q}(K, G)$ into the subgroup $B^{q}(W, G)$ of $Z^{q}\left(W, H_{q}(K), l, G\right)$; this gives the induced homomorphism (13.1). By $\$ 10, N_{e}^{q}$ is contained in the kernel of $\theta$, so that $\Gamma_{\alpha} f=\left(\theta_{f}, r_{f}\right)$ carries $N_{e}^{q}$ into the subgroup $Z^{q}(W, G)$ of $Z^{q}\left(W, H_{q}(K), l, G\right)$. Thus (13.3) may be obtained by "cutting down" the homomorphism $\Gamma_{\alpha}$ of (13.1) to the subgroup $N_{e}^{q}$. Similarly, (13.2) follows by the definition of $\chi$. 
The equation (13.4) asserts that $\Gamma_{\alpha}$ carries the decomposition of $H_{e}^{\alpha}$ (by its subgroup $N_{e}^{q}$ ) into the decomposition of $E^{q}$ (by its subgroup $H^{q}$ ). In fact, for $f \in Z_{e}^{q}(K, G)$,

$$
\left(\chi \Gamma_{\alpha}\right) f=\chi\left(\Gamma_{\alpha} f\right)=\chi\left(\theta_{f}, r_{f}\right)=\theta_{f},
$$

while $\theta \phi f=\theta_{\phi f}=\theta_{f}$, proving (13.4). This also implies that $\chi \Gamma_{\alpha}$ is independent of the choice of $\alpha$. By assumption $H_{q-1}(K)=0$, hence $\theta$ is an isomorphism by Lemma 10.1, and the kernel $N_{e}^{q}(K, G)$ of $\phi$ is the counter image under $\Gamma$ of the kernel $H^{q}(W, G)$ of $\chi$.

To prove that the homomorphism (13.3) is independent of $\alpha$, let $\beta: K_{W}^{q}$ $\rightarrow K^{q}$ be a second chain transformation, with an obstruction $l^{\prime}$. By Theorem 5.1 , there is a homotopy $D: \alpha \simeq \beta(\operatorname{dim} \leqq q-1)$, while $l^{\prime}$ and $l$ are cohomologous, with $l^{\prime}=l+\delta h$ and $h \in C^{q}\left(W, H_{q}(K)\right)$, defined by $h=\eta E_{q}=\eta\left(\beta_{q}-\alpha_{q}\right.$ $\left.-D_{q-1} \partial\right)$, as in the proof of Lemma 4.2. We wish to compare the mappings

$$
\Gamma_{\alpha}: H_{e}^{q}(K, G) \rightarrow E, \quad \Gamma_{\beta}: H_{e}^{q}(K, G) \rightarrow E^{\prime},
$$

where $E=E^{q}\left(W, H_{q}(K), l, G\right)$ and $E^{\prime}=E^{q}\left(W, H_{q}(K), l^{\prime}, G\right)$. Using the isomorphism $\gamma_{h}: E^{\prime} \rightarrow E$ of $\S 12$, we assert that

$$
\gamma_{h} \Gamma_{\beta}=\Gamma_{\alpha}
$$

The definitions of $\Gamma_{\alpha}, \Gamma_{\beta}$, and $\gamma_{h}$ yield, for any $f \in Z_{e}^{q}(K, G)$,

$$
\Gamma_{\alpha} f=\left(\theta_{f}, r_{f}\right), \Gamma_{\beta} f=\left(\theta_{f}, r_{f}^{\prime}\right), \gamma_{h} \Gamma_{\beta} f=\left(\theta_{f}, r_{f}^{\prime}-\theta_{f} h\right),
$$

where $r_{f}=f \alpha_{q}, r_{f}^{\prime}=f \beta_{q}$; hence

$$
\gamma_{h} \Gamma_{\beta} f-\Gamma_{\alpha} f=\left(0, r_{f}^{\prime}-r_{f}-\theta_{f} h\right) .
$$

We have then

$$
r_{f}^{\prime}-r_{f}-\theta_{f} h=f \beta_{q}-f \alpha_{q}-f\left(\beta_{q}-\alpha_{q}-D_{q-1} \partial\right)=f D_{q-1} \partial=\delta\left(f D_{q-1}\right) .
$$

Defining the cochain $s=f D_{q-1} \in C^{q-1}(W, G)$ we have

$$
\gamma_{h} \Gamma_{\beta} f-\Gamma_{\alpha} f=(0, \delta s) \in B^{q}(W, G) .
$$

This proves (13.5).

By Theorem 12.1, $\gamma_{h}$ is the identity on the subgroup $H^{q}(W, G)$ of $E^{q}$; hence (13.5) implies that the cut-down homomorphism $\Gamma_{\alpha}$ of (13.3) is indeed independent of the choice of $\alpha$.

14. The fundamental theorem.

TheOREM 14.1. If $K$ is an augmentable complex with free operators $W$, acyclic in dimensions less than $q$, with characteristic cohomology class $1 \in H^{q+1}\left(W, H_{q}(K)\right)$, then the qth equivariant cohomology group of $K$ with any coefficient group $G$ is determined by the groups $W, H_{q}(K), G$, the way $W$ operates on the latter two groups, and any cocycle $l$ in the class 1 , as 


$$
H_{e}^{q}(K, G) \cong E^{q}\left(W, H_{q}(K), l, G\right) .
$$

The isomorphism is such as to carry $\theta$ into $\chi$, where

$$
\theta: H_{e}^{q} \rightarrow \text { Ophom }\left(H_{q}(K), G\right), \quad \chi: E^{q} \rightarrow \text { Ophom }\left(H_{q}(K), G\right) ;
$$

hence it also carries the subgroup $N_{e}^{q}(K, G)$ of $H_{e}^{q}$ onto the subgroup $H^{q}(W, G)$ of $E^{q}\left({ }^{4}\right)$.

The properties (13.1) and (13.2) of the main homomorphism $\Gamma$ show that this theorem will follow at once if $\Gamma$ is an isomorphism onto; this will be dernonstrated in the next section. A second proof of the theorem, depending directly on Theorem 7.1 , will be given in $\$ 17$.

For lower dimension $p<q$ the homology group $H_{p}$ and hence the corresponding obstruction $l^{p+1}$ vanish, and the group $E^{q}(W, H, l, G)$ becomes simply the $q$ th cohomology group of $W$. Hence the theorem also includes the result previously established in [19] for these lower groups:

$$
H_{e}^{p}(K, G) \cong H^{p}(W, G), \quad \text { for } p<q .
$$

The fundamental theorem, when applied to a topological space, reads as follows:

TheOREm 14.1'. Let $X$ be a topological space acyclic in dimension less than $q(q>0)$. Let $W$ be a multiplicative group operating as a group of transformations on $X$, such that no transformation $w: X \rightarrow X$ has a fixed point for $w \neq 1$. Let $1^{q+1}(X) \in H^{q+1}\left(W, H_{q}(X)\right)$ be the characteristic cohomology class of the singular complex $S(X)$. Then the equivariant cohomology groups $H_{b}^{q}(X, G)$ for any abelian group $G$ with $W$ as (left) operators are determined up to an isomorphism by the groups $W, H_{q}(X), G$, the way $W$ operates on the latter two groups, and by the element $1^{a+1}(X)$. More precisely,

$$
H_{e}^{q}(X, G) \cong E\left(W, H_{q}(X), l, G\right),
$$

where $l$ is any cocycle in the class $I^{a+1}(X)\left({ }^{4}\right)$.

To deduce this theorem from the previous one, we observe that $S(X)$ is an augmentable complex with operators and that, because of Lemma 9.1, $W$ operates freely on $S(X)$.

Under the conditions of Theorem 14.1', the following corollaries hold.

CoRollary 14.2. Under the isomorphism (14.3) the kernel $N_{e}^{q}(X, G)$ of the homomorphism $\phi: H_{e}^{q}(X, G) \rightarrow H^{q}(X, G)$ corresponds to the subgroup $H^{q}(W, G)$ of $E$ :

$$
N_{e}^{q}(X, G) \cong H^{q}(W, G) .
$$

(4) If $q=0$, we employ the homology and cohomology groups of the augmented complex. 
CoRollary 14.3. The quotient group $H_{e}^{q}(X, G) / N_{e}^{q}(X, G)$ is isomorphic with the subgroup $A\left(l^{q+1}(X)\right)$ of Ophom $\left(H_{q}(X), G\right)$ consisting of those operator homomorphisms $\rho: H_{q}(X) \rightarrow G$ with the property that the induced homomorphism $\rho: H^{q+1}\left(W, H_{q}(X)\right) \rightarrow H^{q+1}(W, G)$ annihilates $1^{q+1}(X)$.

Corollary 14.4. If $1^{q+1}(X)=0$, then $N_{e}^{q}(X, G)$ is a direct summand of $H_{e}^{q}(X, G)$ and $H_{e}^{q}(X, G) / N_{e}^{q}(X, G)$ is isomorphic with Ophom $\left(H^{q}(X), G\right)$. Thus, in this case

$$
H_{e}^{q}(X, G) \cong H^{q}(W, G)+\text { Ophom }\left(H_{q}(X), G\right) .
$$

The isomorphism (14.4) was first proved in $[19, \S 16]$. The results for lower dimensional cohomology groups are again included.

15. First proof of the fundamental theorem. We must show that the homomorphism $\Gamma$ of (13.1) is an isomorphism onto, when $W$ operates freely on the complex $K$. Let $\Gamma$ be constructed from the chain transformation $\alpha: K_{W}^{q} \rightarrow K^{q}$ with obstruction $l$. Since $K$ has free operators $W$ and $K_{W}$ is acyclic, there is also a chain transformation $\gamma: K^{q+1} \rightarrow K_{W}^{q+1}$, while by Theorem 5.1 there are chain homotopies

$$
D: \alpha \gamma \simeq I(\operatorname{dim} \leqq q-1), \quad L: \gamma \alpha \simeq I_{W}(\operatorname{dim} \leqq q),
$$

and $E_{q}=I-\alpha_{q} \gamma_{q}-D_{q-1} \partial: C_{q}(K) \rightarrow Z_{q}(K)$, as in (3.6).

To prove $\Gamma$ an isomorphism consider an equivariant cocycle $f \in Z_{\bullet}^{q}(K, G)$ such that $\Gamma f=\left(\theta_{f}, r_{f}\right) \in B^{q}(W, G)$. This means that $\theta_{f}=0$ and that $r_{f}=\delta s$ for some $s \in C^{q-1}(W, G)$. Since $E_{q}$ has cycles as values,

$$
f E_{q}=\theta_{f} \eta E_{q}=0
$$

by the definition of $E_{q}$ and $r_{f}$ this becomes

$$
\begin{aligned}
f & =f \alpha_{q} \gamma_{q}+f D_{q-1} \partial=r_{f} \gamma_{q}+f D_{q-1} \partial=\delta\left(s \gamma_{q}\right)+f D_{q-1} \partial \\
& =\delta\left(s \gamma_{q}+f D_{q-1}\right) .
\end{aligned}
$$

In other words, $f$ is an equivariant coboundary. This proves that $\Gamma$ in (13.1) is an isomorphism into $E^{q}(W, H, l, G)$.

To prove that $\Gamma$ is a mapping onto, consider any element $(\rho, r)$ $\in Z^{q}\left(W, H_{q}(K), l, G\right)$, where, by the definition of the latter group,

$$
\rho \in \text { Ophom }\left(H_{q}(K), G\right), \quad r \in C^{q}(W, G), \quad \delta r=\rho l .
$$

Observe first that

$$
r(z)=\rho \eta \alpha z, \quad \text { for } z \in Z_{q}\left(K_{W}\right) .
$$

In fact, since $K_{W}$ is acyclic, $z=\partial d$ for some $d$ and

$$
r(z)=r(\partial d)=\delta r d=\rho l d=\rho \eta \alpha \partial d=\rho \eta \alpha z .
$$


Next define

$$
h=r-r \gamma_{q} \alpha_{q}-\rho \eta E_{q} \alpha_{q}
$$

and prove

$$
h \in Z^{q}(W, G) .
$$

This $h$ is clearly equivariant, while, for $d \in C_{q+1}\left(K_{W}\right)$,

$$
\begin{aligned}
(\delta h)(d) & =h(\partial d)=r(\partial d)-r(\gamma \alpha \partial d)-\rho \eta E_{q} \alpha \partial d \\
& =r(\partial d)-r(\gamma \alpha \partial d)-\rho \eta \alpha \partial d+\rho \eta \alpha \gamma \alpha \partial d
\end{aligned}
$$

by the definition of $E_{q}$. The result is zero, by (15.1), since both $\partial d$ and $\gamma \alpha \partial d$ are $q$-cycles of $K_{W}$. Hence $\delta h=0$, as asserted in (15.3).

Next define $f \in C^{q}(K, G)$ by

$$
f=\rho \eta E_{q}+r \gamma_{q}+h \gamma_{q} .
$$

Since $\rho, E_{q}, r, \gamma$, and $h$ are equivariant, so is $f$. If $z$ is a cycle, then

$$
\begin{aligned}
f(z) & =\rho \eta(z-\alpha \gamma z)+r(\gamma z)+h(\gamma z) \\
& =\rho \eta z-\rho \eta \alpha \gamma z+r(\gamma z)+h(\gamma z) .
\end{aligned}
$$

Since $\gamma z$ is a cycle in $K_{W}$ and hence also a boundary, while $h$ is a cocycle, we have $h(\gamma z)=0$. Also $r(\gamma z)=\rho \eta \alpha \gamma z$ by $(15.1)$; hence $f z=\rho \eta z$. Thus $\delta f=f \partial$ $=\rho \eta \partial=0$; hence $f$ is a cocycle, and $\theta_{f}=\rho$.

Now we have by (15.4), (15.2), and (15.3),

$$
\begin{aligned}
r_{f} & =f \alpha_{q}=\rho \eta E_{q} \alpha_{q}+r \gamma_{q} \alpha_{q}+h \gamma_{q} \alpha_{q} \\
& =r-h+h \gamma_{q} \alpha_{q} \\
& =r-h\left(I_{q}-\gamma_{q} \alpha_{q}+L_{q-1} \partial\right)-h L_{q-1} \partial \\
& =r-h \partial L_{q}-h L_{q-1} \partial \\
& =r-h L_{q-1} \partial .
\end{aligned}
$$

Hence $r_{f}=r-\delta s$, where $s=h L_{q-1}$, and

$$
\Gamma f=\left(\theta_{f}, r_{f}\right)=(\rho, r-\delta s)=(\rho, r)-(0, \delta s),
$$

so that $\Gamma f \equiv(\rho, r)\left(\bmod B^{q}(W, G)\right)$. This shows that $\Gamma$ is a mapping onto, and completes the proof of the fundamental theorem.

16. Topologized coefficients. It is usually assumed that the coefficient group $G$ used for cohomology is a topological group and that each automorphism $w: G \rightarrow G$ is continuous. In this case the various groups of cochains and cocycles and the various cohomology groups are topologized [19, §3-4]. Similarly, the group Hom $(H, G)$, and its subgroup Ophom $(H, G)$ carry a 
topology $[19, \S 3]$. Consequently the group (12.1) and its subgroup $Z^{q}(W, H, l, G)$ are topological, thus showing that $H^{q}(W, H, l, G)$ is a topological group.

It may be verified that the various homomorphisms defined in this chapter are continuous. In proving Lemma 10.1 and Theorem 14.1 one is also required to prove that the inverses of $\theta$ and $\Gamma$ are continuous. These proofs do not present any difficulty. However, there is a very important case in which these additional proofs are superfluous. That is the case when $G$ is a compact (Hausdorff) group. In this case all the topological groups involved are compact, and the inverse of a 1-1 continuous map is continuous in virtue of a general theorem.

The case of $G$ compact is of special significance because the duality relation between homology and cohomology $[19, \S 6]$

$$
H_{q}^{\bullet}(K, \operatorname{Char} G) \cong \operatorname{Char} H_{e}^{q}(K, G)
$$

allows us to compute the equivariant homology groups.

17. The construction of a complex. The second proof of the fundamental theorem will proceed by the construction of a typical complex with a given obstruction; by Theorem 10.3, the qth cohomology groups of any such complex are isomorphic to those of the typical complex, so that the fundamental theorem can be proved again by showing that $E^{q}$ is the cohomology group of the typical complex.

Let $W$ be a multiplicative group, operating on the left on the (additive) abelian group $H$, and let $l \in Z^{q+1}(W, H)$ be a cocycle. We propose to construct a complex $L=L_{q}(W, l, H)$, acyclic in dimensions less than $q$, having $H_{q}(L) \cong H$, and having $l$ as (one of) its characteristic cocycle(s). We wish $W$ to operate freely on $L$, but the method of construction may be set forth most easily by ignoring this requirement at first.

Let $L_{0}$ be the complex in which the groups of chains are $C_{i}\left(L_{0}\right)=C_{i}\left(K_{W}\right)$ for $i \neq q$ and $C_{q}\left(L_{0}\right)=H+C_{q}\left(K_{W}\right)$, with operators $W$ defined in the natural fashion. The boundary operator $\partial^{\prime}$ is to be the same as the operator $\partial$ in $K_{W}$, except for $q$-chains and $(q+1)$-chains. For a $q$-chain $h+c$, set

$$
\partial^{\prime}(h+c)=\partial c, \quad c \in C_{q}\left(K_{W}\right), h \in H .
$$

For a $(q+1)$-chain $c$, set

$$
\partial^{\prime} c=\partial c-l c, \quad c \in C_{a+1}\left(K_{W}\right) .
$$

Clearly, $\partial^{\prime}$ is an operator homomorphism, and $\partial^{\prime} \partial^{\prime}=0$. The $q$ th homology group is $H_{q}\left(L_{0}\right)=H$, and the identity transformation of $K_{W}$ into $L_{0}$ is a chain transformation $\alpha: K_{W}^{q} \rightarrow L_{0}^{q}$ with obstruction $\eta \alpha \partial c=\eta\left(\partial^{\prime} c+l c\right)=\eta l c=l c$, as intended. The essential step was the addition of the intended homology group $H$ to the group of $q$-chains, plus the insertion of the "twist" by means of $l$ in the boundary formula (17.1). 
It remains to modify $L_{0}$ by replacing $H$ by a group with free operators $W$. By Lemma 2.2, there exists a group $F$ with free operators $W$ and an operator homomorphism $\eta_{0}: F \rightarrow H$ onto $H$. Since $W$ operates freely on $C_{q+1}\left(K_{W}\right)$, the homomorphism $l: C_{q}+1\left(K_{W}\right) \rightarrow H$ can be lifted to an operator homomorphism

$$
l^{*}: C_{q+1}\left(K_{W}\right) \rightarrow F
$$

such that $\eta_{0} l^{*}=l$. If the kernel of $\eta_{0}$ is $R$, there also exists by Lemma 2.2 a group $S$ with free operators $W$ and a homomorphism $\partial_{0}: S \rightarrow R$ onto $R$; the image of $\partial_{0}$ is then the kernel of $\eta_{0}$.

The $(q+1)$-dimensional complex $L$ is to have as its groups of chains the groups

$$
\begin{array}{cc}
C_{q+1}(L)=S+C_{q+1}\left(K_{W}\right), & C_{q}(L)=F+C_{q}\left(K_{W}\right), \\
C_{i}(L)=C_{i}\left(K_{W}\right), & 0 \leqq i<q,
\end{array}
$$

with operators $W$. The boundary operator is to agree with that of $K_{W}$ on chains of dimension less than $q$; for higher dimensions it is

$$
\begin{array}{lrl}
\partial(a, c)=\partial c, & a \in F, c \in C_{q}\left(K_{W}\right), \\
\partial(s, d)=\left(\partial_{0} s-l^{*} d, \partial d\right), & s \in S, d \in C_{q+1}\left(K_{W}\right) .
\end{array}
$$

Thus $\partial$ is an operator homomorphism, and $\partial \partial=0 . L=L(W, l, H)$, as constructed, is an augmentable complex with free operators $W$, acyclic in dimensions less than $q$.

The $q$-cycles in $L$ are the pairs $(a, c)$ with $\partial c=0$. Since $K_{W}$ itself is acyclic in dimension $q$, this implies that $c=\partial d$; hence the $q$-cycles may be written as $(a, \partial d)$. For each such cycle we set

$$
\eta(a, \partial d)=\eta_{0} a+l d, \quad d \in C_{q+1}\left(K_{W}\right) .
$$

Since $l$ is a cocycle, the result here is independent of the choice of $d$ with $\partial d=c$; hence $\eta$ is a homomorphism of $Z_{q}(L)$ onto $H$. By the definition of $l^{*}$ it follows that $\eta$ annihilates boundaries (17.5) in $B_{q}(L)$. Conversely, if $\eta(a, \partial d)=0$, then $\eta_{0} a=-l d=-\eta_{0} l^{*} d$, so that $a+l^{*} d$ lies in the kernel $R$ of $\eta_{0}$, hence in the image $\partial_{0} S$ of $S$, hence has the form $\partial_{0} s$; this makes $(a, \partial d)$ $=\partial(s, d)$ a boundary in $L$, according to the definition (17.5). Consequently $\eta: Z_{q}(L) \rightarrow H$ has kernel $B_{q}(L)$, and therefore provides the natural homomorphism of $Z_{q}(L)$ onto the homology group $H_{q}(L)=H$.

Let $\alpha: K_{W}^{q} \rightarrow L^{q}$ be the obvious chain transformation, with $\alpha_{i}$ the identity for $i<q$ and $\alpha_{q} c=(0, c)$ for any $q$-chain $c$. Its obstruction is

$$
l_{\alpha} d=\eta \alpha_{q} \partial d=\eta(0, \partial d)=l d,
$$

thus is equal to the given cocycle $l$. We conclude that the complex $L$ as constructed has all the desired properties.

A direct analysis now shows that the group $Z^{q}(W, H, l, G)$ constructed 
in $\$ 12$ may be identified with the group $Z_{e}^{q}(L, G)$ of equivariant cocycles on the complex $L$, and that this identification carries the subgroup $B^{q}(W, G)$ of the first group into $B_{e}^{q}(L, G)$. Therefore

$$
E^{q}(W, H, l, G)=H_{e}^{q}(L, G),
$$

and $\chi$ is thereby identified with $\theta$. Theorems 10.3 and 7.1 applied to $L$ thus give a second proof of the fundamental theorem 14.1, independent of the construction of $\Gamma(\$ \S 11,13,15)$. (Note, however, that this proof provides an isomorphism, which would turn out to be equal to $\Gamma$.)

\section{Chapter III. Relations Between homotopy AND homology groups}

18. The universal covering space. As was stated in the Introduction, the results concerning the relations between the homotopy groups and the homology (or rather cohomology) groups of a space $X$ will be obtained by passing to the universal covering space $\tilde{X}$, treated as a space with operators. The requisite results concerning the relations between $X$ and $\tilde{X}$ are summarized in this section. More details may be found in [19, chap. IV].

Let $X$ be an arcwise connected space with homotopy groups $\pi_{i}(X)$ defined relative to some fixed base point $x_{0}$. The group $\pi_{1}(X)$ is written multiplicatively; the groups $\pi_{i}(X)$ for $i>1$ are abelian and are written additively. The group $\pi_{1}(X)$ acts as a group of operators on the groups $\pi_{i}(X)$ for all $i \geqq 1$; in particular, for $i=1$ the element $w \in \pi_{1}$ operates on $s \in \pi_{1}$ as $w(s)=w s w^{-1}$.

There is a natural homomorphism

$$
\nu: \pi_{i}(X) \rightarrow H_{i}(X)
$$

of each homotopy group into the corresponding (integral) homology group (obtained by using finite singular chains). This homomorphism satisfies

$$
\nu(w s)=\nu(s), \quad w \in \pi_{1}, s \in \pi_{i} .
$$

Hence, if we regard $\pi_{1}$ as a group of trivial operators on $H_{i}, \nu$ becomes an operator homomorphism.

The image of $\pi_{i}$ in $H_{i}$ is denoted by $\Sigma_{i}(X)$ and is called the spherical subgroup of $H_{i}(X)$. Cycles belonging to homology classes in $\Sigma_{i}$ are called spherical.

The universal covering space $\tilde{X}$ of $X$ has a natural projection

$$
U: \tilde{X} \rightarrow X
$$

onto $X$ and has the group $\pi_{1}(X)$ as a group of homeomorphisms ("covering transformations") which, except for the identity transformation, have no fixed points, and which operate in such fashion that

$$
U(w x)=U(x) \quad \text { for each } x \in \tilde{X}, w \in \pi_{1}(X) .
$$

Since $\tilde{X}$ is arcwise connected, its homotopy groups may be defined relative 
to some base point $\tilde{x}_{0}$, which may be chosen so that its projection $U\left(\tilde{x}_{0}\right)=x_{0}$ is the given base point in $X$. The transformation $U$ then induces homomorphisms

$$
U: \pi_{i}(\tilde{X}) \rightarrow \pi_{i}(X), \quad i=1, \cdots,
$$

on the homotopy groups. It is known that

$$
\begin{aligned}
\pi_{1}(\tilde{X}) & =0, \\
U: \pi_{i}(\tilde{X}) & \cong \pi_{i}(X), \quad \text { for } i>1 .
\end{aligned}
$$

Since $\pi_{1}(\tilde{X})=0$ by (18.1), the definition of $\pi_{i}(\tilde{X})$ can be made independent of the base point $\tilde{x}_{0}$; from this definition it results that the homeomorphisms $w: \tilde{X} \rightarrow \tilde{X}$ induce automorphisms of $\pi_{i}(\tilde{X})$. Thus $\pi_{1}(X)$ acts on $\pi_{i}(\tilde{X})$ as a group of operators, and it may be shown that the isomorphism (18.2) is an operator isomorphism.

Similarly, $\pi_{1}(X)$ acts as a group of operators on the (singular) homology group $H_{i}(X)$, and $U$ induces an operator homomorphism

$$
U: H_{i}(\tilde{X}) \rightarrow H_{i}(X) \text {. }
$$

In the diagram

$$
\begin{array}{cr}
\pi_{i}(\tilde{X}) & \stackrel{\bullet}{\rightarrow} H_{i}(\tilde{X}) \\
\downarrow U & \downarrow U \\
\pi_{i}(X) \stackrel{\mapsto}{\rightarrow} H_{i}(X)
\end{array}
$$

we have the commutativity relation $U \nu=\nu U$.

LemMA 18.1. If $i>1, \Sigma_{i}(\tilde{X})$ maps on $\Sigma_{i}(X)$ under (18.3).

Proof. Let $x \in \Sigma_{i}(X)$. Then $x=\nu(s)$ for some $s \in \pi_{i}(X)$. Hence $x=\nu U\left(U^{-1} s\right)$ $=U\left(\nu U^{-1} s\right)$, but $\nu U^{-1} \in \Sigma_{i}(\tilde{X})$.

For any abelian coefficient group $G$, the cohomology groups $H^{q}(X, G)$ of the space may be compared with the equivariant cohomology groups $H_{e}^{q}(\tilde{X}, G)$ of the covering space, where in the latter groups we regard $\pi_{1}(X)$ as operating on $\tilde{X}$ by means of covering transformations and on $G$ trivially. Each cochain $f \in C^{q}(X, G)$ has a corresponding equivariant cochain $U^{*} f$ $\in C_{e}^{q}(\tilde{X}, G)$, defined as

$$
\left(U^{*} f\right)(c)=f(U c), \quad \text { for } c \in C_{q}(\tilde{X}) .
$$

The correspondence $f \rightarrow U^{*} f$ induces a homomorphism

$$
U_{\theta}^{*}: H^{q}(X, G) \rightarrow H_{e}^{q}(\tilde{X}, G)
$$

which was proved $[19, \S 25]$ to be an isomorphism onto $H_{e}^{q}$.

A cocycle $f$ in $Z^{q}(X, G)$ is said to be a spherical annihilator if $f(z)=0$ for 
every spherical cycle $z$. The cohomology classes of spherical annihilators constitute a subgroup $\Lambda^{q}(X, G)$ of $H^{q}(X, G)$; clearly, this subgroup $\Lambda^{q}$ is the annihilator of $\Sigma_{q}(X)$ under the homomorphism

$$
\theta: H^{q}(X, G) \rightarrow \operatorname{Hom}\left(H_{q}(X), G\right)
$$

as defined in $\$ 10$. The relation between this subgroup $\Lambda^{q}$ of $H^{q}(X, G)$ and the kernel $N_{e}^{q}(\tilde{X}, G)$ of the homomorphism $\phi: H_{e}^{q}(X, G) \rightarrow H^{q}(\widetilde{X}, G)$ is now to be examined.

Lemma 18.2. $U_{e}^{*} \Lambda^{q}(X, G)=\phi^{-1} \Lambda^{q}(\tilde{X}, G)$.

Proof. Let $f \in Z^{q}(X, G)$ and $z \in Z_{q}(\tilde{X})$. The definition of $U^{*} f$ gives $f(U z)$ $=U^{*} f(z)$. Since by Lemma $18.1, U \Sigma_{q}(\tilde{X})=\Sigma_{q}(X)$, it follows that $f$ is a spherical annihilator in $X$ if and only $U^{*} f$ is in $\tilde{X}$. This is the content of the lemma. then

LEMMA 18.3. If the homomorphism $\pi_{q}(\tilde{X}) \rightarrow H_{q}(\tilde{X})$ is onto, and if $H_{q-1}(\tilde{X})=0$,

$$
U_{e}^{*}: \Lambda^{q}(X, G) \cong N_{\bullet}^{q}(\tilde{X}, G)
$$

is an isomorphism onto.

Proof. Since $\pi_{q}(\tilde{X}) \rightarrow H_{q}(\tilde{X})$ is onto, we have $\Sigma_{q}(\tilde{X})=H_{q}(\tilde{X})$. Thus $f \in \Lambda^{q}(\tilde{X}, G)$ is equivalent with $\theta_{f}=0$. By Lemma 10.1 , we then have $f=0$ and consequently $\Lambda^{q}(\tilde{X}, G)=0$. Lemma 18.2 then yields

$$
U_{e}^{*} \Lambda^{q}(X, G)=\phi^{-1}(0)=N_{e}^{q}(\tilde{X}, G) .
$$

REMARK. The condition $H_{q-1}(\tilde{X})=0$ might be replaced by the weaker requirement that $\theta: H^{q}(\tilde{X}, G) \rightarrow \operatorname{Hom}\left(H_{q}(\tilde{X}), G\right)$ be an isomorphism. The last condition is always fulfilled if $G$ is the group of reals reduced modulo 1 , because in this case $\theta$ is the isomorphism used in the homology-cohomology duality theorem.

19. The characteristic cohomology class of a space. In the remainder of this chapter it will be assumed that the arcwise connected space $X$ is aspherical in dimensions between 1 and $q$, in the sense that

$$
\pi_{i}(X)=0 \quad \text { for } 1<i<q, q>1 .
$$

By (18.2) and (18.3)

$$
\pi_{i}(\tilde{X})=0
$$

for $i<q$,

and applying a well known theorem of Hurewicz [12 ], we find that

$\tilde{X}$ is acyclic in dimensions less than $q$,

$$
\nu: \pi_{q}(\tilde{X}) \cong H_{q}(\tilde{X})
$$


Combining (19.4) with (18.2), we obtain an operator isomorphism

$$
\beta=U \nu^{-1}: H_{q}(\tilde{X}) \cong \pi_{q}(X) .
$$

Now, $\tilde{X}$ is a space acyclic in dimensions less than $q$ and $\pi_{1}(X)$ acts on $\tilde{X}$ as a group of operators. Consequently, the characteristic cohomology class

$$
l^{q+1}(\tilde{X}) \in H^{q+1}\left(\pi_{1}(X), H_{q}(\tilde{X})\right)
$$

is defined. Using the operator isomorphism (19.5), this in turn defines a cohomology class

$$
k^{q+1}(X) \in H^{q+1}\left(\pi_{1}(X), \pi_{q}(X)\right)
$$

In fact, if $l$ is a cocycle in $1^{q+1}(\tilde{X})$, then a cocycle $k$ in $k^{q+1}(X)$ is defined by $k=\beta l$.

In the case when $q=2$, condition (19.1) becomes vacuous. Thus

$$
k^{3}(X) \in H^{3}\left(\pi_{1}(X), \pi_{2}(X)\right)
$$

is defined for any arcwise connected space $X$. An intrinsic definition of $k^{3}(X)$ without the use of $\tilde{X}$ was given in [21] by the authors. We now outline an intrinsic definition of $k^{q+1}(X)$ for any $q$, and sketch the proof that this definition is equivalent to the above definition by the covering space. Fix a base point $x_{0}$ in $X$ and define a function $R$ which for each $n \leqq q$ assigns to each $n$-tuple of elements $w_{1}, \cdots, w_{n}$ of $\pi_{1}(X)$ a singular $n$-simplex $R\left(w_{1}, \cdots, w_{n}\right)$ in $X$, or, more precisely, an equivalence class of singular $n$-simplices in $X$. This function $R$ is subject to two conditions:

(i) For $n=1, R(w)$ is a singular 1 -simplex with both vertices at $x_{0}$ which, when considered as a closed path, represents the element $w$ of $\pi_{1}(X)$.

(ii) For $q \geqq n>1$, the $i$ th face of $R\left(w_{1}, \cdots, w_{n}\right)$ is $R\left(w_{2}, \cdots, w_{n}\right)$ if $i=0$, $R\left(w_{1}, \cdots, w_{i} w_{i+1}, \cdots, w_{n}\right)$ if $0<i<n$, and $R\left(w_{1}, \cdots, w_{n-1}\right)$ if $i=n$.

The definition of $R$ proceeds by an induction with respect to $n$; at each stage the faces of the simplex $R\left(w_{1}, \cdots, w_{n}\right)$ are already determined in consistent fashion, and the interior can be "filled in" because of the assumption that $\pi_{n-1}(X)=0$.

Given $R$, consider a $(q+1)$-tuple $w_{1}, \cdots, w_{q+1}$ of elements of $\pi_{1}(X)$. Let $\Delta$ be an Euclidean $(q+1)$-simplex with ordered vertices $v_{0}<\cdots<v_{q+1}$, and let $\dot{\Delta}$ be its boundary. By mapping the faces $\Delta^{(0)}, \cdots, \Delta^{(q+1)}$ of $\Delta$ into $X$ according to the maps

$$
R\left(w_{2}, \cdots, w_{q+1}\right), \cdots, R\left(w_{1}, \cdots, w_{i} w_{i+1}, \cdots, w_{q+1}\right), \cdots, R\left(w_{1}, \cdots, w_{q}\right),
$$

we obtain a map

$$
\dot{\Delta} \rightarrow X
$$

By treating $\dot{\Delta}$ as a $q$-sphere with a base point $v_{0}$, this map determines an element 


$$
k^{q+1}\left(w_{1}, \cdots, w_{n+1}\right) \in \pi_{q}(X) .
$$

This function $k^{q+1}$ is the obstruction to the continuation of the definition of $R$. We treat $k^{q+1}$ as a nonhomogeneous (see $\$ 26$ below) $(q+1)$-cochain $k^{q+1}$ $\in C^{q+1}\left(\pi_{1}(X), \pi_{q}(X)\right)$. It is easy to see that $k^{q+1}$ is a cocycle and that its cohomology class does not depend on the choice of $R$. The cohomology class $\boldsymbol{k}^{q+1}(X) \in H^{q+1}\left(\pi_{1}(X), \pi_{q}(X)\right)$ thus obtained is called the characteristic class of $X$. It may be shown that any cocycle $k^{q+1}$ in the class $k^{q+1}$ can be obtained by a suitable choice of $R$.

To see that this definition of $k^{q+1}(X)$ is in accordance with the definition utilizing the universal covering space $\tilde{X}$, we select a base point $\tilde{x}_{0} \in \tilde{X}$ such that $U \tilde{x}_{0}=x_{0}$, and "lift" every singular simplex $R\left(w_{1}, \cdots, w_{n}\right)$ in $X$ to a singular simplex $\tilde{R}\left(w_{1}, \cdots, w_{n}\right)$ in $X$ such that $U \tilde{R}=R$ and that $\tilde{x}_{0}$ is the leading vertex of $R\left(w_{1}, \cdots, w_{n}\right)$. For each $i=0, \cdots, q$ the function $\widetilde{R}\left(w_{1}, \cdots, w_{i}\right)$ defines a (nonhomogeneous) cochain $f^{i} \in C^{i}\left(\pi_{1}(X), C_{i}(\tilde{X})\right)$. This sequence of cochains satisfies the conditions of Theorem 6.3 and therefore may be used to define an obstruction cocycle $l^{q+1}$ in the characteristic cohomology class $1^{q+1}(\tilde{X}) \in H^{q+1}\left(\pi_{1}(X), H_{q}(\tilde{X})\right)$, where $\tilde{X}$ is treated as a space with the group $\pi_{1}(X)$ as operators. It is apparent that the cocycles

$$
k^{q+1} \in Z^{q+1}\left(\pi_{1}(X), \pi_{q}(X)\right), \quad l^{q+1} \in Z^{q+1}\left(\pi_{1}(X), H_{n}(\tilde{X})\right)
$$

defined by $R$ and $\widetilde{R}$ respectively are related by the isomorphism $\beta=U \nu^{-1}$ of the coefficient groups.

20. Computation of the cohomology groups. We come now to the main results of this chapter. Of this theorem only relation (20.2) was known [6, Theorem II].

TheOREM 20.1. Let $X$ be an arcwise connected topological space such that

$$
\pi_{i}(X)=0 \quad \text { for } 1<i<q, q>1 .
$$

Then the cohomology group $H^{q}(X, G)$ is determined by the homotopy groups $\pi_{1}(X)$ and $\pi_{q}(X)$, the operators of $\pi_{1}$ on $\pi_{q}$, the coefficient group $G$, and the characteristic cohomology class $k^{q+1}(X) \in H^{q+1}\left(\pi_{1}, \pi_{q}\right)$. More precisely

$$
H^{q}(X, G) \cong E^{q}\left(\pi_{1}, \pi_{q}, k, G\right),
$$

where $k$ is any cocycle in $k^{q+1}(X)$, and $E^{q}$ is the group constructed in $\$ 12$ (where $\pi_{1}$ is assumed to operate trivially on $G$ ). For the subgroup $\Lambda^{q}$ of spherical annihilators the isomorphism (20.1) induces isomorphisms

$$
\begin{gathered}
\Lambda^{q}(X, G) \cong H^{q}\left(\pi_{1}, G\right), \\
H^{q}(X, G) / \Lambda^{q}(X, G) \cong A\left(\boldsymbol{k}^{q+1}(X)\right),
\end{gathered}
$$

where $A\left(k^{q+1}(X)\right)$ is the group of those operator homomorphisms of $\pi_{q}$ into $G$ for which the induced homomorphism $H^{q+1}\left(\pi_{1}, \pi_{q}\right) \rightarrow H^{q+1}\left(\pi_{1}, G\right)$ annihilates $k^{q+1}(X)$. In particular, if $k^{q+1}(X)=0$, then $\Lambda^{q}(X, G)$ is a direct summand of 
$H^{q}(X, G)$, the group $A\left(k^{q+1}(X)\right)$ coincides with the group Ophom $\left(\pi_{q}, G\right)$ of all operator homomorphisms, and $H^{q}(X, G)$ is a direct sum

$$
H^{q}(X, G) \cong \text { Ophom }\left(\pi_{q}, G\right)+H^{q}\left(\pi_{1}, G\right) .
$$

Proof. Consider the universal covering space $\tilde{X}$ of $X$ and the isomorphism $\beta: H_{q}(\tilde{X}) \cong \pi_{q}(X)$. Let $k$ be a cocycle in the characteristic cohomology class $k^{q+1}(X) \in H^{q+1}\left(\pi_{1}, \pi_{q}(X)\right)$. Then $l=\beta^{-1} k$ is in the cohomology class $1^{q+1}(\widetilde{X})$ $\in H^{q+1}\left(\pi_{1}, H_{q}(\tilde{X})\right)$.

We consider the isomorphism

$$
U_{e}^{*}: H^{q}(X, G) \cong H_{e}^{q}(\tilde{X}, G) .
$$

Since $\tilde{X}$ satisfies the conditions of Theorem 14.1', we also have an isomorphism

$$
\Gamma: H_{e}^{q}(\tilde{X}, G) \cong E^{q}\left(\pi_{1}, H_{q}(\tilde{X}), l, G\right) .
$$

Furthermore, an application of $\beta$ gives an isomorphism

$$
E^{q}\left(\pi_{1}, H_{q}(\tilde{X}), l, G\right) \cong E^{q}\left(\pi_{1}, \pi_{q}, k, G\right) .
$$

The composition of the isomorphisms (20.5)-(20.7) yields (20.1).

Now we observe that because of (19.3) and (19.4) the conditions of Lemma 18.3 are met, and therefore

$$
U_{e}^{*}: \Lambda^{q}(X, G) \cong N_{\bullet}^{q}(\tilde{X}, G) .
$$

By Corollary 14.2,

$$
\Gamma: N_{e}^{q}(\tilde{X}, G) \cong H^{q}\left(\pi_{1}, G\right) .
$$

Furthermore, we observe that $H^{q}\left(\pi_{1}, G\right)$ is a subgroup of both the groups occurring in (20.7) and that this isomorphism leaves $H^{q}\left(\pi_{1}, G\right)$ pointwise fixed, hence (20.2) follows. The remainder of the theorem follows from Corollaries 14.3 and 14.4 .

In the special case when $q=2$ (discussed in [21]), the condition $\pi_{i}(X)=0$ for $1<i<q$ becomes vacuous and Theorem 20.1 applies to any arcwise connected space, to give

$$
H^{2}(X, G) \cong E^{2}\left(\pi_{1}, \pi_{2}, k, G\right)
$$

where $k \in k^{3}(X) \in H^{3}\left(\pi_{1}, \pi_{2}\right)$.

21. The reduced form of Theorem 20.1. The previous discussion did not take advantage of the fact that the operators of $\pi_{1}(X)$ on the group $G$ were simple. Taking notice of this fact allows us to put Theorem 20.1 in a somewhat stronger form. Since the transition is pure algebraic, we return to the group $E^{q}(W, H, l, G)$ discussed in $\$ 12$ and assume that $W$ operates simply on $G$. Let $H_{0}$ denote the subgroup of $H$ spanned by the elements $h-w h, h \in H$, 
$w \in W$. We introduce the factor group $H / H_{0}$ and the natural homomorphism

$$
\omega: H \rightarrow H_{r}=H / H_{0}
$$

with kernel $H_{0}$. If $W$ is allowed to operate on $H_{r}$ simply, then $\omega$ is an operator homomorphism. Since the operators of $W$ on $G$ are simple, every operator homomorphism $H \rightarrow G$ has the form $\rho \omega$ where $\rho: H_{r} \rightarrow G$ is an ordinary homomorphism.

If $(\rho \omega, r)$ is an element of $Z^{q}(W, H, l, G)$ for $\rho \in \operatorname{Hom}\left(H_{r}, G\right), r \in C^{q}(W, G)$, then $\delta r=\rho \omega l$, so that $(\rho, r)$ is an element of $Z^{q}\left(W, H_{r}, \omega l, G\right)$ where $\omega l \in Z^{q+1}\left(W, H_{r}\right)$. The correspondence $(\rho \omega, r) \rightarrow(\rho, r)$ yields an isomorphism

$$
\Omega: Z^{q}(W, H, l, G) \cong Z^{q}\left(W, H_{r}, \omega l, G\right) .
$$

This isomorphism leaves the common subgroup $Z^{q}(W, G)$ pointwise fixed and induces an isomorphism

$$
\Omega: E^{q}(W, H, l, G) \cong E^{q}\left(W, H_{r}, \omega l, G\right) .
$$

In order to apply this isomorphism $\Omega$ to the group occurring in Theorem 20.1 , we replace the groups $W$ and $H$ by the groups $\pi_{1}(X)$ and $\pi_{q}(X)$, and define the groups $\pi_{q, 0}(X), \pi_{q, r}(X)=\pi_{q} / \pi_{q, 0}$ and the homomorphism $\omega: \pi_{q} \rightarrow \pi_{q, r}$ as above. The group $\pi_{q, r}$ will be called the reduced $q$ th homotopy group of $X$. The homomorphism $\omega$ induces a homomorphism

$$
H^{q+1}\left(\pi_{1}, \pi_{q}\right) \rightarrow H^{q+1}\left(\pi_{1}, \pi_{q, r}\right) .
$$

The image of the characteristic cohomology class $k^{q+1}(X)$ is denoted by $\boldsymbol{k}_{r}^{q+1}(X)$ and is called the reduced characteristic cohomology class of $X$. Theorem 20.1 may now be restated as follows.

THEOREM 21.1. Let $X$ be an arcwise connected topological space such that

$$
\pi_{i}(X)=0 \quad \text { for } 1<i<q, q>1 .
$$

Then the cohomology group $H^{a}(X, G)$ is determined by the groups $\pi_{1}(X)$, $\pi_{q, r}(X), G$, and by the reduced cohomology class $k_{r}^{q+1}(X) \in H^{q+1}\left(\pi_{1}, \pi_{q, r}\right)$. More precisely,

$$
H^{q}(X, G) \cong E^{q}\left(\pi_{1}, \pi_{q, r}, k_{r}, G\right),
$$

where $k_{r}$ is any cocycle in $k_{r}^{a+1}(X)$, and $\pi_{1}$ is assumed to operate simply both on $\pi_{Q, r}$ and $G$.

This isomorphism induces isomorphisms

$$
\begin{gathered}
\Lambda^{q}(X, G) \cong H^{q}\left(\pi_{1}, G\right), \\
H^{q}(X, G) / \Lambda^{q}(X, G) \cong A\left(k_{r}^{q+1}(X)\right),
\end{gathered}
$$

where $A\left(k_{r}^{a+1}(X)\right)$ is the subgroup of Hom $\left(\pi_{q, r}, G\right)$ consisting of those homo- 
morphisms such that the induced homomorphism $H^{q+1}\left(\pi_{1}, \pi_{q, r}\right) \rightarrow H^{q+1}\left(\pi_{1}, G\right)$ annihilates $\boldsymbol{k}^{q+1}(X)$.

If ${k_{r}^{a+1}}^{a+}(X)=0$, then $\Lambda^{q}(X, G)$ is a direct summand of $H^{q}(X, G)$, the group $A\left(k_{r}^{q+1}(X)\right)$ coincides with $\operatorname{Hom}\left(\pi_{q, r}, G\right)$, and $H^{q}(X, G)$ is a direct sum

$$
H^{q}(X, G) \cong \operatorname{Hom}\left(\pi_{q, r}, G\right)+H^{q}\left(\pi_{1}, G\right) \text {. }
$$

Proof. Let $k$ be any cocycle in the cohomology class $\boldsymbol{k}^{q+1}(X)$. Theorem 20.1 then yields

$$
H^{q}(X, G) \cong E^{q}\left(\pi_{1}, \pi_{q}, k, G\right),
$$

while the isomorphism $\Omega$ defined above yields

$$
E^{q}\left(\pi_{1}, \pi_{q}, k, G\right) \cong E^{q}\left(\pi_{1}, \pi_{q, r}, \omega k, G\right) .
$$

Since both $k_{r}$ and $\omega k$ are in the cohomology class $k_{r}^{a+1}(X)$, there is a cochain $h \in C^{q}\left(\pi_{1}, \pi_{q, r}\right)$ with $\delta h=\omega k-k_{r}$. Then $\gamma_{h}$ defined in $\S 12$ is an isomorphism

$$
E^{q}\left(\pi_{1}, \pi_{q, r}, \omega k, G\right) \cong E^{q}\left(\pi_{1}, \pi_{q, r}, k_{r}, G\right) .
$$

The composition of (21.5)-(21.7) implies (21.1). Observe now that both (21.6) and (21.7) leave the subgroup $H^{q}\left(\pi_{1}, G\right)$ pointwise fixed. Thus (20.2) implies (21.2). Next notice that (21.6) induces an isomorphism

$$
A\left(k^{q+1}(X) \cong A\left(k_{r}^{q+1}(X)\right),\right.
$$

while (21.7) leaves $A\left(k_{r}^{a+1}(X)\right)$ pointwise fixed. Thus (21.3) is a consequence of (20.3).

It may be worth noting that the isomorphism (21.8) is induced by the isomorphism

$$
\text { Ophom }\left(\pi_{q}, G\right) \cong \operatorname{Hom}\left(\pi_{q, r}, G\right),
$$

obtained by factoring out the map $\omega$.

22. Local coefficients. The passage from $\pi_{q}$ to $\pi_{q, r}$ and from $k^{q+1}(X)$ to $k_{r}^{a+1}(X)$ in the previous section was possible only because $\pi_{1}$ operated trivially on $G$. This is no longer the case if we consider groups with local coeffcients $[17],[19$, chap. V].

Let $\left\{G_{x}\right\}$ be a local system of groups in the arcwise connected space $X$. Let $G=G_{x 0}$, where $x_{0}$ is the base point in $X$. Then $\pi_{1}(X)$ operates on $G$.

We consider the cohomology group $H^{q}\left(X,\left\{G_{x}\right\}\right)$ with $\left\{G_{x}\right\}$ as local coefficient system. The analogue of (18.4) takes then the form

$$
U_{e}^{*}: H^{q}\left(X,\left\{G_{x}\right\}\right) \cong H_{e}^{q}(\tilde{X}, G)
$$

Following the suggestion of Lemma 18.2, the subgroup $\Lambda^{q}\left(X,\left\{G_{x}\right\}\right)$ of $H^{q}\left(X,\left\{G_{x}\right\}\right)$ is defined as

$$
\Lambda^{q}\left(X,\left\{G_{x}\right\}\right)=U_{e}^{*-1} \phi^{-1} \Lambda^{q}(\tilde{X}, G),
$$


and Lemma 18.3 still holds.

With these definitions, the analogue of Theorem 20.1 takes the following form:

Theorem 22.1. If $\pi_{i}(X)=0$ for $1<i<q, q>1$, then

$$
\begin{gathered}
H^{q}\left(X,\left\{G_{x}\right\}\right) \cong E^{q}\left(\pi_{1}, \pi_{q}, k, G\right), \\
\Lambda^{q}\left(X,\left\{G_{x}\right\}\right) \cong H^{q}\left(\pi_{1}, G\right), \\
H^{q}\left(X,\left\{G_{x}\right\}\right) / \Lambda^{q}\left(X,\left\{G_{x}\right\}\right) \cong A\left(k^{q+1}(X)\right),
\end{gathered}
$$

where $k \in k^{q+1}(X) \in H^{q+1}\left(\pi_{1}, \pi_{q}\right)$.

If $\boldsymbol{k}^{q+1}(X)=0$, then $\Lambda^{q}\left(X,\left\{G_{x}\right\}\right)$ is a direct summand of $H^{q}\left(X,\left\{G_{x}\right\}\right)$ and $A\left(k^{q+1}(X)\right)=$ Ophom $\left(\pi_{q}, G\right)$ so that $H^{q}\left(X,\left\{G_{x}\right\}\right)$ is a direct sum:

$$
H^{q}\left(X,\left\{G_{x}\right\}\right) \cong \text { Ophom }\left(\pi_{q}, G\right)+H^{q}\left(\pi_{1}, G\right) .
$$

Formula (22.4) has been proved before $[19, \S 28]$.

23. The integral homology groups. An application of the character theory of Pontrjagin allows us to dualize Theorem 20.1 and derive a similar theorem for the $q$ th homology groups of $X$. Now we shall formulate these results, but only for the integral homology group $H_{q}(X)$. Consequently, we shall choose the coefficient group $G$ for the cohomology groups to be the additive group $P$ of reals reduced mod 1. The groups appearing in (21.1)-(21.3) are then compact, and the isomorphisms are topological.

Let $f$ be a cohomology class in $H^{q}(X, P) . \theta_{f}$ is a homomorphism $\theta_{f}: H_{q}(X)$ $\rightarrow P$, and thus $\theta_{f}$ is an element of the character group Char $H_{q}(X)$. It is known that this correspondence of $f$ to $\theta_{f}$ is an isomorphism. If we identify $f$ and $\theta_{f}$, then

$$
H^{q}(X, P)=\text { Char } H_{q}(X)
$$

and therefore

$$
H_{g}(X)=\operatorname{Char} H^{q}(X, P) .
$$

Applying this to (21.1), we find that, under the conditions of Theorem 21.1,

$$
H_{q}(X) \cong \operatorname{Char} E^{q}\left(\pi_{1}, \pi_{q, r}, k_{r}, P\right) .
$$

Now, by definition, $\Lambda^{q}(X, P)$ is the annihilator of the subgroup $\Sigma_{q}(X)$ of $H_{g}(X)$. Consequently, it follows from (21.2) that the subgroup $H^{q}\left(\pi_{1}, P\right)$ is the annihilator of $\Sigma_{q}(X)$ under (23.1). Standard results in character theory then give

$$
\begin{aligned}
H_{q}(X) / \Sigma_{q}(X) & \cong \operatorname{Char} H^{q}\left(\pi_{1}, P\right)=H_{q}\left(\pi_{1}\right), \\
\Sigma_{q}(X) & \cong \operatorname{Char}\left(E^{q} / H^{q}\left(\pi_{1}, P\right)=\operatorname{Char}\left(A\left(k_{r}^{q+1}(X)\right),\right.\right.
\end{aligned}
$$

where $H_{q}\left(\pi_{1}\right)$ is the integral homology group of $\pi_{1}$, while $A\left(k_{r}^{q+1}(X)\right)$ 
$=E^{q} / H^{q}\left(\pi_{1}, P\right)$ is the subgroup of the group Hom $\left(\pi_{q, r}, P\right)=$ Char $\pi_{q, r}$, consisting of those homomorphisms $\rho: \pi_{q, r} \rightarrow P$ such that the induced homomorphism $H^{q}\left(\pi_{1}, \pi_{q, r}\right) \rightarrow H^{q}\left(\pi_{1}, P\right)$ annihilates the cohomology class $k_{r}^{q+1}(X)$. Because $A\left(k_{r}^{q+1}(X)\right)$ is a subgroup of Char $\pi_{q, r}$, the group Annih $A\left(k_{r}^{q+1}(X)\right)$ is a subgroup of $\pi_{q, r}$; thus, by (23.3)

$$
\Sigma_{q}(X) \cong \pi_{q . r}(X) / \text { Annih } A\left(k_{r}^{q+1}(X)\right) .
$$

Suppose, in particular, that $k_{r}^{q+1}(X)=0$. Then $A\left(k_{r}^{q+1}\right)$ coincides with Hom $\left(\pi_{q, r}, P\right)$, its annihilator is zero, and hence by $(23.4) \Sigma_{q}(X) \cong \pi_{q, r}(X)$. In addition, $\Lambda^{q}(X, P)$ is a direct summand of $H^{q}(X, P)$, from which it results that $\Sigma_{q}(X)=$ Annih $\Lambda^{q}(X, P)$ is a direct summand of $H_{q}(X)$. Consequently,

$$
H_{q}(X)=\pi_{q, r}(X)+H_{q}\left(\pi_{1}(X)\right), \quad \text { if } k_{r}^{q+1}(X)=0 .
$$

Formula (23.4) suggests that the group Annih $A\left(k_{\tau}^{q+1}(X)\right)$ is the kernel of the homomorphism $\pi_{q, r} \rightarrow H_{q}$. We proceed to discuss this point in more detail. Since $\nu: \pi_{q} \rightarrow H_{q}$ is an operator homomorphism and since $\pi_{1}$ operates simply on $H_{q}$, there is a homomorphism

$$
\nu_{r}: \pi_{q, r}(X) \rightarrow H_{q}(X),
$$

such that $\nu=\nu_{r} \omega$, where $\omega$ is the natural map $\omega: \pi_{q} \rightarrow \pi_{q} / \pi_{q, 0}=\pi_{q, r}$. We study the kernels of $\nu$ and $\nu_{r}$; since $\nu$ carries $\pi_{q, 0}$ into 0 , it is clear that the kernel of $\nu$ contains $\pi_{q, 0}$, and has the kernel of $\nu_{r}$ as its factor group, modulo $\pi_{q, 0}$. The kernel of $\nu_{r}$ was studied by Hopf [11], who denoted it by $\Delta^{q}$.

TheOREM 23.1. Let $X$ be an arcwise connected space for which $\pi_{i}(X)=0$, for $1<i<q, q>1$. The kernel of $\nu: \pi_{q}(X) \rightarrow H_{q}(X)$ is then Annih $A\left(k^{q+1}(X)\right)$, where $A\left(k^{q+1}(X)\right)$ consists of those operator homomorphisms $\rho: \pi_{q} \rightarrow P$ for which the induced homomorphism $H^{q+1}\left(\pi_{1}, \pi_{q}\right) \rightarrow H^{q+1}\left(\pi_{1}, P\right)$ annihilates $k^{q+1}(X)$. The kernel of $\nu_{r}: \pi_{q, r}(X) \rightarrow H_{q}(X)$ is Annih $A\left(k_{r}^{q+1}(X)\right)$. In particular, $\nu_{r}$ is an isomorphism into if and only if $A\left(k_{r}^{q+1}(X)\right)=$ Char $\pi_{q, r}$. Consequently, if $k_{r}^{q+1}(X)=0$, then $\nu_{r}$ has kernel zero, or equivalently

$$
\nu: \pi_{q}(X) \rightarrow \Sigma_{q}(X)
$$

has kernel $\pi_{q, 0}(X)$.

Proof. Let $s \in \pi_{q}(X)$ and let $z$ be the corresponding element of $H_{q}(\tilde{X})$ under the isomorphism $\beta$ of (19.5). It follows from the diagram in $\$ 18$ that

$$
\nu s=U z .
$$

If $f \in H^{q}(X, P)$, then $U_{e}^{*} f=h \in H_{e}^{q}(\tilde{X}, P)$, and

$$
(\theta f)(\nu s)=(\theta f)(U z)=(\theta h)(z)=(\theta \phi h)(z) .
$$

We now appeal to the diagram 


$$
\begin{array}{cc}
H_{e}^{q}(\tilde{X}, P) \stackrel{\Gamma}{\rightarrow} E\left(\pi_{1}, H_{q}(\tilde{X}), l, P\right) \\
\quad \downarrow \phi & \downarrow \chi \\
H^{q}(\tilde{X}, P) \stackrel{\theta}{\rightarrow} \operatorname{Hom}\left(H_{q}(\tilde{X}), P\right) .
\end{array}
$$

By (13.4) of Lemma (13.1), $\theta \phi=\chi \Gamma$, so that we have

$$
(\theta f)(\nu s)=(\theta \phi h)(z)=(\chi \Gamma h)(z) \text {. }
$$

In order that

$$
\nu s=0,
$$

it is necessary and sufficient that

$$
(\theta f)(\nu s)=0 \quad \text { for all } f \in H^{q}(X, P) .
$$

As $f$ ranges over all of $H^{q}(X, P)$, the element $h$ ranges over all of $H_{\theta}^{q}(\tilde{X}, P)$, $\Gamma h$ ranges over all of $E^{q}$ and $\rho=\chi \Gamma h$ ranges over all of $A\left(1^{q+1}(\tilde{X})\right)$, for the latter group is the image of $E$ under $\chi$. Hence (23.6) is equivalent with

$$
\rho(z)=0 \quad \text { for all } \rho \in A\left(1^{q+1}(\tilde{X})\right),
$$

and if we apply (19.5) again, we find that (23.6) is equivalent with

$$
\rho^{\prime}(s)=0 \quad \text { for all } \rho^{\prime} \in A\left(k^{q+1}(X)\right) .
$$

This proves the first half of the theorem.

To prove the second half of the theorem, consider an element $s \in \pi_{q}$. Since $\nu s=\nu_{r} \omega s$, it follows by the first part of the theorem, that $\nu_{r}(\omega s)=0$ if and only if

$$
s \in \operatorname{Annih}\left(A\left(k^{q+1}(X)\right) .\right.
$$

Now let $\rho \in A\left(k^{q+1}(X)\right) \subset$ Ophom $\left(\pi_{q}, P\right)$. Then $\rho=\rho_{r} \omega$ for a suitable $\rho_{r} \in$ Hom $\left(\pi_{q, r}, P\right)$. Since

$$
\rho k^{q+1}=\rho_{r} \omega k^{q+1}=\rho_{r} k_{r}^{q+1},
$$

the correspondence $\rho \rightarrow \rho_{r}$ carries $A\left(k^{q+1}(X)\right)$ isomorphically onto $A\left(k_{r}^{q+1}(X)\right)$. Since

$$
\rho(s)=\rho_{r}(\omega s),
$$

it follows that (23.7) is equivalent with

$$
\omega s \in \text { Annih }\left(A\left(k_{r}^{q+1}(X)\right) .\right.
$$

This concludes the proof.

24. Computation of examples. Let $X$ be an arcwise connected space such that $\pi_{i}(X)=0$ for $1<i<q, q>1$. If, in addition, $k_{r}^{a+1}(X)=0$, then the results of $\$ 23$ show that 


$$
\begin{aligned}
& \Sigma_{q}(X) \text { is a direct summand of } H_{q}(X) ; \\
& \nu: \pi_{q}(X) \rightarrow \Sigma_{q}(X) \text { has kernel } \pi_{q, 0}(X) .
\end{aligned}
$$

These two facts can be used to prove that $k_{r}^{q+1}(X) \neq 0$ for a large number of spaces. This a fortior $i$ implies $k^{a+1}(X) \neq 0$.

For example, let $X$ be the $q$-dimensional projective space. The group $\pi_{1}(X)$ is then cyclic of order 2 , and $\pi_{i}(X)=0$ for $1<i<q$. If $q$ is odd, then $H_{q}(X)$ is cyclic infinite, while $\Sigma_{q}(X)$ consists of all the elements of $H_{q}(X)$ which are divisible by 2 . Thus $(24.1)$ implies that $k_{r}^{q+1}(X) \neq 0$. If $q$ is even, then $H_{q}(X)=0$, and $\Sigma_{q}(X)=0$. On the other hand $\pi_{q}(X)$ is cyclic infinite while $\pi_{q, 0}(X)$ is a proper subgroup of $\pi_{q}(X)$. Thus (24.2) implies that $k_{r}^{q+1}(X)$ $\neq 0$.

It follows that $k^{q+1}(X) \neq 0$, if $X$ is a $q$-dimensional projective space. Now consider the group

$$
H^{q+1}\left(\pi_{1}(X), \pi_{q}(X)\right),
$$

in which $k^{q+1}(X)$ lies. $\pi_{1}$ is cyclic of order 2 while $\pi_{q}$ is cyclic infinite. If $q$ is odd, then $\pi_{1}$ operates simply on $\pi_{q}$ and $H^{q+1}\left(\pi_{1}, \pi_{q}\right)$ is cyclic of order 2 by $[7, \$ 16]$ or $\$ 26$ below. If $q$ is even, then the element different from 1 in $\pi_{1}$ operates on $\pi_{q}$ by transforming each element into its inverse, and again $H^{q+1}\left(\pi_{1}, \pi_{q}\right)$ is cyclic of order 2 by $[7, \S 16]$. Thus the group (24.3) is cyclic of order 2 for all $q$, so that the fact that $k^{q+1}(X) \neq 0$ actually gives a computation of the invariant.

In the case of the projective spaces, a direct computation of $k^{q+1}(X)$ is not too difficult.

Another method for obtaining information about $k_{r}^{q+1}(X)$ is by an application of a theorem of Hopf [11], which asserts that if, in addition to the conditions listed above, $X$ is a $q$-dimensional polyhedron, then the kernel of $\nu_{r}$ is isomorphic to

$$
H_{q+1}\left(\pi_{1}(X)\right)=\text { Char } H^{q+1}\left(\pi_{1}(X), P\right) .
$$

Hence, if $H^{q+1}\left(\pi_{1}(X), P\right) \neq 0$, the kernel of $\nu_{r}$ is not zero, and $k_{r}^{q+1}(X) \neq 0$.

\section{Chapter IV. Rotations AND LENS SPACES}

25. Rotations. Let the surface $S^{2 n+1}$ of the unit sphere in an even dimensional Euclidean space $R^{2 n+2}$ be given in terms of $n+1$ complex coordinates $z_{0}, \cdots, z_{n}$ by the equation

$$
\sum_{i=0}^{n}\left|z_{i}\right|^{2}=1
$$

Choose a positive integer $h$ and $n+1$ integers $l_{0}, \cdots, l_{n}$ relatively prime to $h$ and define the primitive $h$ th roots of unity $\rho_{i}$ by the equations 


$$
\rho_{i}^{l_{i}}=e^{2 \pi i / h}, \quad i=0, \cdots, n ;\left(l_{i}, h\right)=1 .
$$

(The $l_{i}$ are determined only $\bmod h$.) Define the rotation of $S^{2 n+1}$ by the equation

$$
T\left(z_{0}, \cdots, z_{n}\right)=\left(\rho_{0} z_{0}, \cdots, \rho_{n} z_{n}\right) .
$$

Then $T$ is a rotation of $S^{2 n+1}$ with period $h$, such that none of the powers $T, T^{2}, \cdots, T^{h-1}$ has a fixed point on $S^{2 n+1}$. Conversely, it may be shown that any rotation of $S^{2 n+1}$ of period $h$ which possesses the last mentioned property may be represented in the form above, for a suitable choice of coordinate system and of integers $l_{i}$.

Let $\Gamma$ designate the (multiplicative) group of all powers of $T$, so that $\Gamma$ is cyclic of order $h$, and $\gamma=T$ is a generator. The space $S^{2 n+1}$ is thus one on which a group operates without fixed points, and hence serves to illustrate our theory. Since $S^{2 n+1}$ is acyclic in dimensions less than $2 n+1$, the characteristic cohomology class

$$
I^{2 n+1}\left(S^{2 n+1}\right) \in H^{2 n+2}\left(\Gamma, H_{2 n+1}\left(S^{2 n+1}\right)\right)
$$

is defined. The coefficient group $H_{2 n+1}\left(S^{2 n+1}\right)$ which appears here is cyclic infinite; the choice of an orientation for the sphere consists in the selection of one of the two possible isomorphisms

$$
H_{2 n+1}\left(S^{2 n+1}\right) \cong J
$$

with the additive group $J$ of integers. Since $T$ acts on an odd-dimensional sphere and has no fixed points, it follows that $T$ preserves the orientation; that is, maps $H_{2 n+1}\left(S^{2 n+1}\right)$ identically upon itself. Hence $\Gamma$ operates trivially on $H_{2 n+1}\left(S^{2 n+1}\right)$. Upon identification of the two groups in (25.2) by means of the given isomorphism, the characteristic cohomology class (25.1) becomes

$$
I^{2 n+2}\left(S^{2 n+1}\right) \in H^{2 n+2}(\Gamma, J)
$$

with $\Gamma$ operating trivially upon $J$.

The sphere $S^{2 n+1}$ with its group $\Gamma$ of transformations may also be regarded as a covering space for a generalized lens space (or "cyclic space," in the terminology of de Rham [24]) $L^{2 n+1}$. This space $L^{2 n+1}$ is obtained by identifying all the images $x, T(x), \cdots, T^{h-1}(x)$ on each point $x$ of $S^{2 n+1}$. It is an orientable manifold, and manifestly has $S^{2 n+1}$ as its universal covering space, with $\Gamma$ as the corresponding group of covering transformations, so that $\Gamma$ is the fundamental group of $L^{2 n+1}$. The homotopy groups $\pi_{i}\left(L^{2 n+1}\right)$ for $1<i<2 n+1$ are zero because the corresponding homology groups of the covering space vanish, and the homotopy group $\pi_{2 n+1}\left(L^{2 n+1}\right)$ may be identified with $H_{2 n+1}\left(S^{2 n+1}\right)=J$. Hence the characteristic cohomology class $k^{2 n+2}\left(L^{2 n+1}\right)$ of the lens space is defined and lies in the group:

$$
k^{2 n+2}\left(L^{2 n+1}\right) \in H^{2 n+2}\left(\pi_{1}\left(L^{2 n+1}\right), \pi_{2 n+1}\left(L^{2 n+1}\right)\right)=H^{2 n+2}(\Gamma, J) .
$$


It is identical with (25.3), in virtue of the results of Chapter III.

The computation of the elements (25.3) and (25.4) is the chief objective of the following sections, which begin with an analysis of the group $H^{2 n+2}(\Gamma, J)$.

26. The group $H^{2 n+2}(\Gamma, J)$. To describe the invariant of $S^{2 n+1}$, it is convenient to use the nonhomogeneous form of the cohomology groups and cocycles of a group $W$, operating on the left on the abelian group $G$. We summarize the description of these cochains, following [7]. An $m$-dimensional nonhomogeneous cochain $f$ of $W$ in $G$ is a function $f\left(x_{1}, \cdots, x_{m}\right)$ of $m$ variables $x_{i}$ in $W$, with values in $G$. The normalization argument of [7, Chap. I], or the equivalent use of the normalized complex $K_{W}^{*}$ of $\S 8$ above, shows that we can restrict the attention to normalized cochains; that is, to cochains $f$ with $f\left(x_{1}, \cdots, x_{m}\right)=0$ whenever any one argument $x_{1}$ is the identity element of $W$. The coboundary of $f$ is a normalized $(m+1)$-dimensional cochain $\delta f$ defined as

$$
\begin{aligned}
& \delta f\left(x_{1}, \cdots, x_{m+1}\right)=x_{1} f\left(x_{2}, \cdots, x_{m+1}\right) \\
& \quad+\sum_{i=1}^{m}(-1)^{i} f\left(x_{1}, \cdots, x_{i} x_{i+1}, \cdots, x_{m+1}\right)+(-1)^{m+1} f\left(x_{1}, \cdots, x_{m}\right),
\end{aligned}
$$

for any $m+1$ arguments $x_{1}, \cdots, x_{m+1}$ in $W$. An $m$-cocycle is an $f$ with $\delta f=0$, an $m$-coboundary, an $f$ of the form $f=\delta h$ for some $(m-1)$-cochain $h$. One has always $\delta \delta f=0$ : hence the additive group of $m$-cocycles contains the group of $m$-coboundaries. It was proved in [7] that the factor group of nonhomogeneous $m$-cocycles modulo $m$-coboundaries is isomorphic to the $m$ th cohomology group $H^{m}(W, G)$, defined as above as the $m$ th equivariant cohomology group of the complex $K_{W}$. The present cochains $f$ are called nonhomogeneous in contrast to the homogeneous cochains, which are simply the equivariant cochains of this complex.

The group $W$ may also be regarded as a group of trivial operators on the additive group $J$ of integers. The groups $G$ and $J$ are paired to $G$, in the sense that $g \in G$ and $n \in J$ determine $g \cup n=n g=g+\cdots+g$ ( $n$ times); this pairing is bilinear and satisfies $x(g \cup n)=(x g) \cup_{n}$ for any $x \in W$. An $m$-cochain $f$ of $W$ in $G$ and a $k$-cochain $g$ of $W$ in $J$ then determine a "cup product" $f \cup g$, which is an $m+k$ cochain of $W$ in $G$, as

(26.2) $(f \cup g)\left(x_{1}, \cdots, x_{m+k}\right)=f\left(x_{1}, \cdots, x_{m}\right) \cup g\left(x_{m+1}, \cdots, x_{m+k}\right)$.

As in $[7, \S 4]$ one has

$$
\delta(f \cup g)=(\delta f) \cup g+(-1)^{m} f \cup \delta g ;
$$

consequently, the cup product of two cocycles is a cocycle, and the cup product induces a (bilinear) cup product between the cohomology classes in $H^{m}(W, G)$ and $H^{k}(W, J)$ with values in $H^{m+k}(W, G)$. In particular, we may use such cup products when $G=J$, and $W$ operates simply on $J$. 
For a cyclic group $\Gamma$ of order $h$ operating trivially on $J$ the even dimensional cohomology groups $H^{2 n+2}(\Gamma, J)$ are isomorphic to $J / h J$, and hence are cyclic of order $h$. The result follows at once by the reduction theorem of $[7$, Chap. III]. The characteristic cohomology class (25.4) of the lens space $L^{2 n+1}$ lies in the group $H^{2 n+2}$, and for the subsequent discussion we need a more explicit form of the isomorphism $H^{2 n+2} \cong J / h J$. This form may be obtained readily, using the nonhomogeneous cochains.

Suppose that $\Gamma$ is cyclic of order $h$ with generator $\gamma$. A special nonhomogeneous 2-cochain of $\Gamma$ in $J$ may be defined by setting, for $0 \leqq i<h, 0 \leqq j<h$,

$$
\begin{array}{ll}
s_{\gamma}^{2}\left(\gamma^{i}, \gamma^{j}\right)=0, & \text { if } i+j<h, \\
s_{\gamma}^{2}\left(\gamma^{i}, \gamma^{j}\right)=1, & \text { if } i+j \geqq h .
\end{array}
$$

That $s_{\gamma}^{2}$ is a cocycle may be shown by direct calculation, using a suitable subdivision into cases, or by realizing $s_{\gamma}^{2}$ as a factor set of the group $J$, regarded as a group extension of the subgroup $h J \cong J$ by $J / h J \cong \Gamma$. Using the cup product, we may then define higher dimensional cocycles as cup products

$$
s_{\gamma}^{2 n+2}=s_{\gamma}^{2} \cup \ldots \cup s_{\gamma}^{2} \quad(n+1 \text { times }) .
$$

TheOREm 26.1. For $n \geqq 0$, the cochain $s_{\gamma}^{2 n+2}$ is a cocycle, and its cohomology class $\mathrm{s}_{\gamma}^{2 n+2}$ is a generator for the group $H^{2 n+2}(\Gamma, J)$, which is cyclic of order $h$. If $\alpha$ is another generator for $\Gamma$, then $\alpha^{t}=\gamma$ some integer $t$ relatively prime to $h$, and

$$
s_{\alpha}^{2 n+2}=t^{n+1} s_{\gamma}^{2 n+2} \text {. }
$$

This theorem can be proved by representing $\Gamma \cong J / h J$ as a factor group of the free group $J$, and then applying to this representation the reduction theorem of [7, Chap. III]. Alternatively, a direct proof can be given for the case of cyclic groups. The direct proof will be presented elsewhere.

27. Formulation of results. With the aid of the preceding section we are now able to give an explicit form for the characteristic cohomology class (25.3) of $S^{2 n+1}$ as

$$
l^{2 n+2}\left(S^{2 n+1}\right)=l_{0}^{-1} \cdots l_{n}^{-1} s_{\gamma}^{2 n+2} \in H^{2 n+2}(\Gamma, J),
$$

where $l_{i}^{-1}$ are integers such that $l_{i} l_{i}^{-1} \equiv 1(\bmod h)$. This is the main result of this chapter. The proof of (27.1) will be given in the following section. In the meantime we shall draw some consequences from (27.1).

Let $S^{\prime 2 n+1}$ be another oriented sphere and $T^{\prime}$ a rotation of $S^{2 n+1}$ of the type described above, with period $h$ and associated integers $l_{0}^{\prime}, \cdots, l_{n}^{\prime}$. The rotations $T$ of $S^{2 n+1}$ and $T^{\prime}$ of $S^{2 n+1}$ are called homeomorphic if there is a homeomorphism $A: S^{2 n+1} \rightarrow S^{\prime 2 n+1}$ such that $T^{\prime} A=A T$. Moreover we shall say that $T$ and $T^{\prime}$ are positively (negatively) homeomorphic if $A$ preserves (reverses) the orientation of $S^{2 n+1}$. We may regard the same cyclic group $\Gamma$ 
as operating on both $S^{2 n+1}$ and $S^{\prime 2 n+1}$, with the generator $\gamma$ of $\Gamma$ operating on $S^{2 n+1}$ as $T$ and on $S^{\prime 2 n+1}$ as $T^{\prime}$. The condition $T^{\prime} A=A T$ then becomes $\gamma A=A \gamma$, which means that the map $A: S^{2 n+1} \rightarrow S^{2 n+1}$ is equivariant. The map $A$ induces a homomorphism

$$
H_{2 n+1}\left(S^{2 n+1}\right) \rightarrow H_{2 n+1}\left(S^{2 n+1}\right),
$$

which in turn induces a h@momorphism

$$
H^{2 n+2}\left(\Gamma, H_{2 n+1}\left(S^{2 n+1}\right)\right) \rightarrow H^{2 n+2}\left(\Gamma, H_{2 n+1}\left(S^{2 n+1}\right)\right),
$$

and under this homomorphism $I^{2 n+2}\left(S^{\prime 2 n+1}\right)$ is the image of $I^{2 n+2}\left(S^{2 n+1}\right)$. In our discussion both groups in (27.2) are identified with the group $J$ and the map (27.2) is the identity of $A$ is a positive homeomorphism or changes the sign of each element if $A$ is a negative homeomorphism. Consequently, we have the following theorem.

THEOREM 27.1. If $T$ is a rotation of $S^{2 n+1}$ of period $h$ with associated integers $l_{0}^{\prime}, \cdots, l_{n}^{\prime}$, then

$$
l_{0} \cdots l_{n} \equiv l_{0}^{\prime} \cdots l_{n}^{\prime}(\bmod h),
$$

if $T$ and $T^{\prime}$ are positively homeomorphic, and

$$
l_{0} \cdots l_{n} \equiv-l_{0}^{\prime} \cdots l_{n}^{\prime}(\bmod h),
$$

if $T$ and $T^{\prime}$ are negatively homeomorphic.

If $L^{2 n+1}$ is a generalized lens space, given in terms of a generator $\gamma$ of its fundamental group and the integers $l_{0}, \cdots, l_{n}$, the main result (27.1) shows that its characteristic cohomology class is

$$
k^{2 n+2}\left(L^{2 n+1}\right)=l_{0}^{-1} \cdots l_{n}^{-1} s_{\gamma}^{2 n+2} .
$$

Note that the formula changes appropriately if $\gamma$ is replaced by $\alpha$, with $\alpha^{t}=\gamma$, $(t, h)=1$, and $l_{i}$ is replaced by the corresponding integer $m_{i}$, defined with respect to $\alpha$, with $m_{i}=t l_{i}$. Let $L^{\prime 2 n+1}$ be another such lens space with integers $l_{0}^{\prime}, \cdots, l_{n}^{\prime}$. Let $H: L^{2 n+1} \rightarrow L^{\prime 2 n+1}$ be a homeomorphism. With the homeomorphism $H$ is associated a pair of integers $(t, e)$ such that $H$ maps the generator of $\pi_{1}\left(L^{2 n+1}\right)$ into $t$ times the generator of $\pi_{1}\left(L^{\prime 2 n+1}\right)$, while $e= \pm 1$, depending whether $H$ maps $L^{2 n+1}$ on $L^{\prime 2 n+1}$ positively or not. It follows from the preceding arguments that

$$
t^{n+1} l_{0} \cdots l_{n} \equiv e l_{0}^{\prime} \cdots l_{n}^{\prime}(\bmod h) .
$$

Thus we have the following theorem.

THEOREM 27.2. In order that two lens spaces $L^{2 n+1}$ and $L^{2 n+1}$ both with fundamental groups cyclic of order $h$ and corresponding to the sets of integers $l_{0}, \cdots, l_{n}$ 
and $l_{0}^{\prime}, \cdots, l_{n}^{\prime}$ respectively be homeomorphic, it is necessary that

$$
t^{n+1} l_{0} \cdots l_{n} \equiv e l_{0}^{\prime} \cdots l_{n}^{\prime}(\bmod h),
$$

where $t$ is an integer relatively prime to $h$ and $e= \pm 1$.

These results were obtained by de Rham [24] and by P. A. Smith [26], using completely different methods.

28. Calculation of the characteristic cocycle of a lens space. The proof of (27.1) requires a suitable cell decomposition of the sphere $S^{2 n+1}$ with operators $\Gamma$. Following De Rham [24] and Franz [25] we introduce in $S^{2 n+1}$ the $h(2 n+1)$-cells defined in terms of the complex coordinates of $\S 25$ as

$$
a_{j}^{2 n+1}: 2 \pi j / h<\arg z_{n}<2 \pi(j+1) / h,
$$

and the $h 2 n$-cells defined by

$$
a_{j}^{2 n}: \arg z_{n}=2 \pi j / h \text {. }
$$

These cells fill all of $S^{2 n+1}$ except for the $2 n-1$ sphere cut out by $z_{n}=0$; this sphere is similarly subdivided. Clearly $\partial a_{j}^{2 n+1}=a_{j+1}^{2 n}-a_{j}^{2 n}$, and $\partial a_{j}^{2 n}$ is the sum of all the $2 n-1$ cells. The action of $\Gamma$ on these cells is found by observing that $\alpha_{n}=\gamma^{l_{n}}$ increases the argument of $z_{n}$ by $2 \pi / h$; hence

$$
\alpha_{n} a_{j}^{2 n+1}=a_{j+1}^{2 n+1}, \quad \alpha_{n} a_{j}^{2 n}=a_{j+1}^{2 n} .
$$

In general, in each dimension $i=0, \cdots, 2 n+1$ there are exactly $h i$-cells, all of which can be obtained from a single $i$-cell $a^{i}$ by application of the transformations of $\Gamma$; the boundary relations are

$$
\begin{aligned}
\partial a^{2 i+1} & =\alpha_{i} a^{2 i}-a^{2 i}, \quad i=0, \cdots, n, \alpha_{i}=\gamma^{l_{i} .} \\
\partial a^{2 i} & =a^{2 i-1}+\gamma a^{2 i-1}+\cdots+\gamma^{h-1} a^{2 i-1}, \quad i=1, \cdots, n .
\end{aligned}
$$

Furthermore, the orientation of $a^{2 n+1}$ agrees with that of $S^{2 n+1}$. Let $K$ denote the (augmentable) complex with operators $\Gamma$ constructed from these cells with the indicated relations. The invariance of the characteristic cohomology class of a space ( $\$ 9)$ shows that the invariant $l^{2 n+2}\left(S^{2 n+1}\right)$ of the sphere with operators may be computed as the invariant $l^{2 n+2}(K)$ of the complex $K$. This latter invariant will be constructed, using a sequence of cocycles as in Theorem 6.3; we may assume that these cocycles are in nonhomogeneous form.

The $i$-cells of $K$ are the cells $z_{1} a^{i}, \cdots, z_{h} a^{i}$, where $z_{1}, \cdots, z_{h}$ are the $h$ elements of the group $\Gamma$, in any order. Hence each integral $i$-chain $c_{i}$ of $K$ may be written as

$$
c_{i}=m_{1} z_{1} a^{i}+\cdots+m_{h} z_{h} a^{i}=r a^{i},
$$

where the coefficient $r=m_{1} z_{1}+\cdots+m_{h} z_{h}$ is any linear combination of the 
symbols $z_{i}$ with integral coefficients $m_{i}$. Thus $r$ is any element in the group ring $R$ of $\Gamma$ over the ring of integers, and the group $C_{i}(K)$ is isomorphic to the additive group of $R$. The multiplication in $R$ is given by the distributive law and the rule $(m x)(n y)=(m n)(x y)$; if $z_{1}$ is the identity of $\Gamma, 1_{R}=1 z_{1}$ is the unit element of $R$ and may be denoted by 1 when there is no danger of confusion. We also set

$$
\sigma=1+\gamma+\cdots+\gamma^{h-1}=z_{1}+\cdots+z_{h}
$$

for the sum of all the elements of $\Gamma$, taken in $R$. Clearly $R$ is a ring of left operators on the group $C_{i}(K)$ of integral $i$-chains in $K$, and the boundary relations (28.1) and (28.2) may be written in the form

$$
\partial a^{2 i+1}=\left(\alpha_{i}-1\right) a^{2 i}, \quad \partial a^{2 i}=\sigma a^{2 i-1} .
$$

The group $\Gamma$ acts as a group of left operators on the additive group of $R$, according to the definition

$$
y\left(\sum_{i} m_{i} z_{i}\right)=\sum_{i} m_{i}\left(y z_{i}\right), \quad y \in \Gamma .
$$

Since the group $J$ of integers appears as the subgroup of elements $m=m 1_{R}$ in the additive group of $R$, multiplication in $R$ provides a pairing $r \cup m=r\left(m 1_{R}\right)$ of $R$ and $J$ to $R$. This pairing is preserved under left operation, for $y(r \cup m)$ $=(y r) \cup m$. We may thus consider nonhomogeneous cochains of $\Gamma$ over $R$ and the cup product of such cochains with cochains of $\Gamma$ over $J$. Furthermore, each $r \in R$ operates on any cochain $f \in C^{q}(\Gamma, R)$ to give the cochain $r f \in C^{q}(\Gamma, R)$ defined as

$$
(r f)\left(x_{1}, \cdots, x_{q}\right)=r\left[f\left(x_{1}, \cdots, x_{q}\right)\right] ; \quad x_{1}, \cdots, x_{q} \in \Gamma .
$$

Each element $r \in R$ may be regarded as a 0 -cochain $\{r\}$ in $C^{0}(\Gamma, R)$. In particular, the element $\sigma \in R$ of (28.3) is invariant under operators $y \in \Gamma$, so that $\{\sigma\}$ is a 0 -cocycle.

For each generator $\alpha$ of $\Gamma$ introduce a 1-cochain $f_{\alpha}^{1} \in C^{1}(\Gamma, R)$ by the definition

$$
f_{\alpha}^{1}\left(\alpha^{i}\right)=\alpha^{0}+\cdots+\alpha^{i-1}, \quad 0 \leqq i<h
$$

Since

$$
\delta\{1\}\left(\alpha^{i}\right)=\alpha^{i}-1=(\alpha-1) \sum_{j=0}^{i-1} \alpha^{i}=(\alpha-1) f_{\alpha}^{1}\left(\alpha^{i}\right)
$$

we have

$$
\delta\{1\}=(\alpha-1) f_{\alpha}^{1} .
$$

Furthermore, for $0 \leqq i<h, 0 \leqq j<h$, we calculate that 


$$
\begin{array}{rlrl}
\delta f_{\alpha}^{1}\left(\alpha^{i}, \alpha^{j}\right) & =0 & & (\text { if } i+j<h) \\
& =\alpha^{i+j-h}+\cdots+\alpha^{i+j-1}=\sigma \quad & \text { (if } i+j \geqq h) .
\end{array}
$$

Hence, using the 2-cocycle $s_{\alpha}^{2}$ of $\$ 26$,

$$
\delta f_{\alpha}^{1}=\{\sigma\} \cup s_{\alpha}^{2}
$$

Use the generators $\alpha_{i}=\gamma^{l_{i}}$ to define cochains $g^{i} \in C^{i}(\Gamma, R), i=0, \cdots, 2 n$ +1 , as

$$
\begin{array}{rlrl}
g^{0} & =\{1\}, \quad g^{1}=f_{\alpha_{0},}^{1} & \\
g^{2 i} & =\{1\} \cup s_{\alpha_{i-1}}^{2} \cup \cdots \cup s_{\alpha_{0},}^{2} & & i=1, \cdots, n, \\
g^{2 i+1} & =f_{\alpha_{i}}^{1} \cup s_{\alpha_{i-1}}^{2} \cup \cdots \cup s_{\alpha_{0}}^{2}, & & i=1, \cdots, n,
\end{array}
$$

and a cochain $h^{2 n+2} \in C^{2 n+2}(\Gamma, J)$ as

$$
h^{2 n+2}=s_{\alpha_{n}}^{2} \cup \cdots \cup s_{\alpha_{0}}^{2}
$$

To compute the successive coboundaries, observe by (26.1) that $\delta\left(h_{1} \cup h_{2}\right)$ $=\left(\delta h_{1}\right) \cup h_{2}$, if $h_{2}$ is a cocycle; hence the rules (28.5) and (28.6) give at once

$$
\begin{aligned}
& \delta g^{2 i}=\left(\alpha_{i}-1\right) g^{2 i+1}, \quad \delta g^{2 i+1}=\sigma g^{2 i+2}, \quad i=0, \cdots, n-1 \text {, } \\
& \delta g^{2 n}=\left(\alpha_{n}-1\right) g^{2 n+1}, \quad \delta g^{2 n+1}=\{\sigma\} \cup h^{2 n+2} .
\end{aligned}
$$

Now construct a sequence of cochains $f^{j} \in C^{j}\left(\Gamma, C_{j}(K)\right)$ for the complex $K$ by setting

$$
f^{j}\left(x_{1}, \cdots, x_{j}\right)=g^{j}\left(x_{1}, \cdots, x_{j}\right) a^{j}, \quad j=0, \cdots, 2 n+1,
$$

where $a^{j}$ is the basic $j$-cell of $K$. The rules (28.4) and (28.7) together now give

$$
\begin{aligned}
\delta f^{j} & =\partial f^{j+1}, \quad j=0,1, \cdots, 2 n+1 ; \\
J f^{0}(1) & =J g^{0}(1) a^{0}=J a^{0}=1 .
\end{aligned}
$$

Hence the $f^{j}$ form a sequence of cochains of the type considered in Theorem 6.3. The obstruction $l$ lies in $H^{2 n+2}\left(\Gamma, H_{2 n+1}(K)\right)$. To consider $H_{2 n+1}(K)$, observe that $\left(\alpha_{n}-1\right) \sigma=0$ in $R$, so that $\sigma a^{2 n+1}$ is by $(28.4)$ a $(2 n+1)$-cycle of $K$. All cycles are multiples of this one, so that the homomorphism $\eta\left(\sigma a^{2 n+1}\right)=1$ identifies $H_{2 n+1}(K)$ with the group $J$ and gives an obstruction $l$ in $H^{2 n+2}(\Gamma, J)$. The obstruction (as a cocycle in $Z^{2 n+2}(\Gamma, J)$ ) is

$$
\begin{aligned}
l^{2 n+2} & =\eta \delta f^{2 n+1}=\eta\left[\delta g^{2 n+1} a^{2 n+1}\right]=\eta\left[\left(\{\sigma\} \cup h^{2 n+2}\right) a^{2 n+1}\right] \\
& =h^{2 n+2} \eta\left(\sigma a^{2 n+1}\right)=h^{2 n+2} \\
& =s_{\alpha_{n}}^{2} \cup \cdots \cup s_{\alpha_{0} \cdot}^{2}
\end{aligned}
$$

Passing to the cohomology classes, and using $\alpha_{i}=\gamma^{l_{i}}$ or $\gamma=\alpha_{i}^{l_{i}-1}$, where 
$l_{i}^{-1} l_{i} \equiv 1(\bmod h)$, we have $s_{\alpha_{i}}^{2}=l_{i}^{-1} s_{\gamma}^{2}$ by Theorem 26.1 , and hence

$$
l^{2 n+2}=l_{0}^{-1} \cdots l_{n}^{-1} s_{\gamma}^{2 n+2} \text {. }
$$

This is the desired formula (27.1)

\section{BIBLIOGRAPHY}

References 1-17 are to the correspondingly numbered items in the Bibliography of part I of this paper ([19] below).

18. S. Eilenberg, Extension and classification of continuous mappings, Lectures in Topology, The University of Michigan Conference of 1940, Ann Arbor, 1941, pp. 57-99.

19. —-, Homology of spaces with operators. I, Trans. Amer. Math. Soc. vol. 61 (1947) pp. 378-417.

20. S. Eilenberg and S. MacLane, General theory of natural equivalences, Trans. Amer. Math. Soc. vol. 58 (1945) pp. 231-294.

21. - Determination of the second homology and cohomology groups of a space by means of homotopy invariants, Proc. Nat. Acad. Sci. U.S.A. vol. 32 (1946) pp. 277-280.

22. W. Franz, Ueber die Torsion einer Überdeckung, J. Reine Angew. Math. vol. 173 (1935) pp. $245-254$.

23. K. Reidemeister, Homotopieringe und Lensentäume, Abh. Math. Sem. Hansischen Univ. vol. 11 (1936) pp. 102-109.

24 G. de Rham, Sur les nouveaux invariants topologiques de M. Reidemeister, Rec. Math. (Mat. Sbornik) vol. 43 (1936) pp. 737-743.

25. H. Robbins, On the classification of the mappings of a 2-complex, Trans. Amer. Math. Soc. vol. 49 (1941) pp. 308-324.

26. P. A. Smith, Periodic and nearly periodic transformations, Lectures on Topology, The University of Michigan Conference of 1940, Ann Arbor, 1941, pp. 159-190.

27. B. Eckmann, On complexes over a ring and restricted cohomology groups, Proc. Nat. Acad. Sci. U.S.A. vol. 33 (1947) pp. 275-281.

COLUMBIA UNIVERSITY, NEW York, N. Y.

UNIVERSITY OF ChICAGo, Chicago, Ill. 